\title{
Targeted superparamagnetic iron oxide nanoparticles for early detection of cancer: Possibilities and challenges
}

\author{
Zahra Bakhtiary, PharmD ${ }^{\mathrm{a}}$, Amir Ata Saei, PharmD ${ }^{\mathrm{b}}$, Mohammad J. Hajipour, $\mathrm{PhD}^{\mathrm{c}}$, \\ Mohammad Raoufi, $\mathrm{PhD}^{\mathrm{d}, \mathrm{f}}$, Ophir Vermesh, $\mathrm{PhD}^{\mathrm{e}}$, Morteza Mahmoudi, $\mathrm{PhD}^{\mathrm{f}, \mathrm{g}, \mathrm{h}, *}$ \\ ${ }^{a}$ Research Center for Pharmaceutical Nanotechnology, Faculty of Pharmacy, Tabriz University of Medical Sciences, Tabriz, Iran \\ ${ }^{\mathrm{b}}$ Department of Medical Biochemistry and Biophysics, Karolinska Institutet, Stockholm, Sweden \\ ${ }^{\mathrm{c}}$ Persian Gulf Marine Biotechnology Research Center, The Persian Gulf Biomedical Sciences Research Institute, \\ Bushehr University of Medical Sciences, Bushehr, Iran \\ ${ }^{\mathrm{d}}$ Department of New Materials and Biosystems, Max Planck Institute for Intelligent Systems, Stuttgart, Germany \\ e Molecular Imaging Program at Stanford (MIPS), Department of Radiology, Stanford University, CA, USA \\ ${ }_{\mathrm{f}}$ Department of Nanotechnology \& Nanotechnology Research Center, Faculty of Pharmacy, Tehran University of Medical Sciences, Tehran, Iran \\ ${ }^{\mathrm{g}}$ Division of Cardiovascular Medicine, Stanford University School of Medicine, Stanford, CA, USA \\ ${ }^{\mathrm{h}}$ Cardiovascular Institute, Stanford University School of Medicine, Stanford, CA, USA \\ Received 4 August 2015; accepted 25 October 2015
}

\begin{abstract}
Nanomedicine, the integration of nanotechnological tools in medicine demonstrated promising potential to revolutionize the diagnosis and treatment of various human health conditions. Nanoparticles (NPs) have shown much promise in diagnostics of cancer, especially since they can accommodate targeting molecules on their surface, which search for specific tumor cell receptors upon injection into the blood stream. This concentrates the NPs in the desired tumor location. Furthermore, such receptor-specific targeting may be exploited for detection of potential metastases in an early stage. Some NPs, such as superparamagnetic iron oxide NPs (SPIONs), are also compatible with magnetic resonance imaging (MRI), which makes their clinical translation and application rather easy and accessible for tumor imaging purposes. Furthermore, multifunctional and/or theranostic NPs can be used for simultaneous imaging of cancer and drug delivery. In this review article, we will specifically focus on the application of SPIONs in early detection and imaging of major cancer types.
\end{abstract}

From the Clinical Editor: Super-paramagnetic iron oxide nanoparticles (SPIONs) have been reported by many to be useful as an MRI contrast agent in the detection of tumors. To further enhance the tumor imaging, SPIONs can be coupled with tumor targeting motifs. In this article, the authors performed a comprehensive review on the current status of using targeted SPIONS in tumor detection and also the potential hurdles to overcome.

(C) 2015 Elsevier Inc. All rights reserved.

Key words: Diagnosis; Ligand; Magnetic resonance imaging; SPION; Targeting

In the United States alone, 1,658,370 new cancer cases and 589,430 cancer deaths have been projected for $2015 .{ }^{1}$ A significant improvement in cancer survival has been noted over the past three decades for most cancer types. This notable and continuous decrement in tumor fatality is related to advanced development in prevention, early diagnosis and therapeutic approaches and

Conflict of interest: Authors declare no conflict of interest.

* Corresponding author.

E-mail addresses: Mahmoudi@stanford.edu,mahmoudi-m@tums.ac.ir (M. Mahmoudi). decrease in overall prevalence of smoking. ${ }^{1}$ Early detection of cancer, especially before cancer cells metastasize, is critical for cancer diagnosis, because early stage cancers can be treated more effectively. However, early-stage diagnosis has been difficult to achieve in most cases since clinical symptoms tend to show up at later stages. Therefore, minimally- and non-invasive methods for early detection of cancer are urgently needed in the field. In this regard, many SPION-based systems are under development for more sensitive imaging and earlier detection of tumors. Since non-targeted SPION species cannot readily differentiate cancer tissues from normal tissues, second-generation SPIONs have been developed which are equipped with targeting moieties that can recognize 


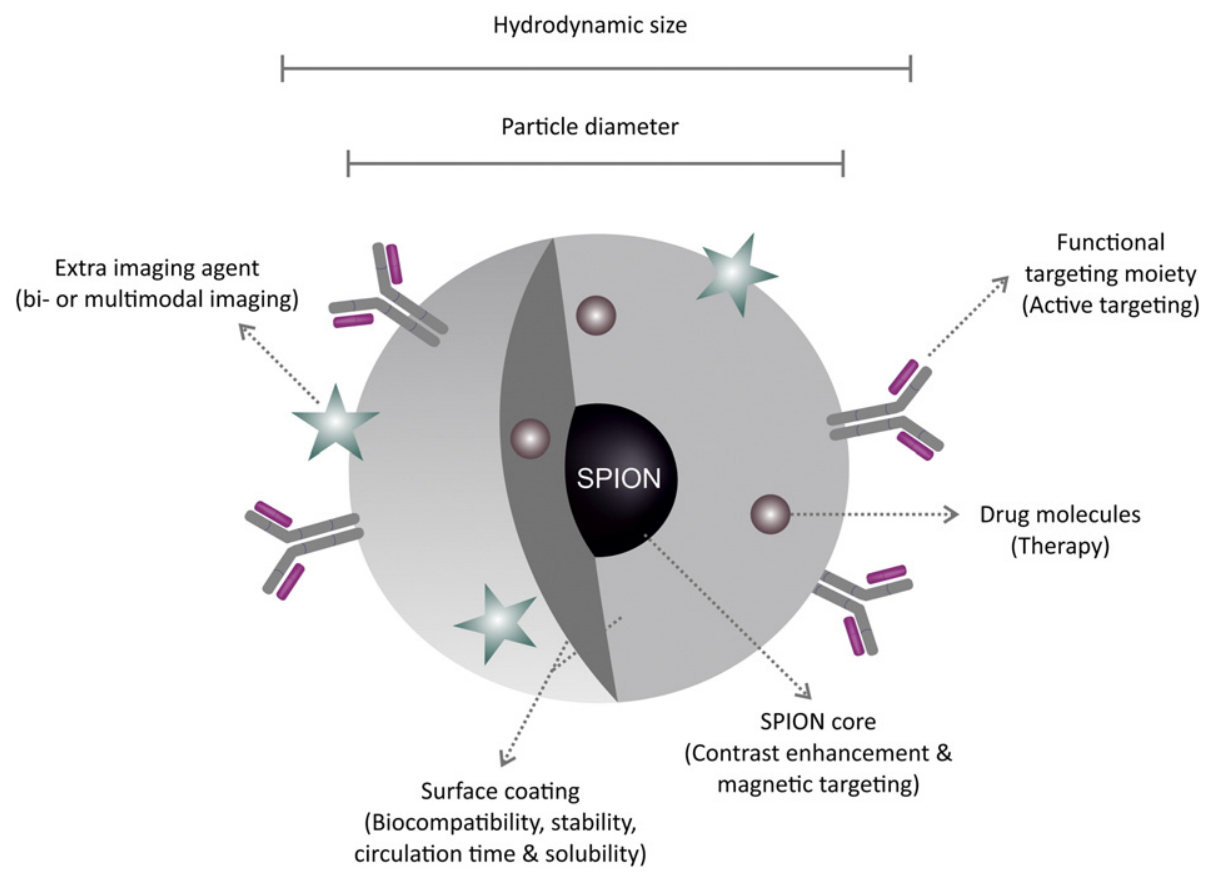

Figure 1. The functions of different components in a SPION-based MRI contrast agent.

specific cell-surface receptors on a given cancer cell type or even subtype. This review provides a concise overview of second-generation targeted SPIONs in the detection and diagnosis of cancer. We will also review the potential challenges associated with development of such platforms and the opportunities that might be provided.

\section{SPIONs as contrast agents}

Unlike other imaging modalities such as X-ray computed tomography (CT) and positron emission tomography (PET), MRI is preferred because it circumvents the need to use ionizing radiation which can cause serious complications upon repeated scanning, especially in children and young adults. ${ }^{2}$ MRI is a non-invasive technique that provides even higher resolution than the former methods. ${ }^{3}$ SPIONs are among the most favored MRI contrast agents. They are also used for drug/gene delivery, ${ }^{4}$ magnetic separation (e.g., in rapid DNA sequencing), ${ }^{5}$ magnetic hyperthermia therapy for cancer, ${ }^{6}$ magnetotransfection, ${ }^{7}$ and stem cell tracking. ${ }^{8}$ This extensive list of biomedical applications is not only attributed to their magnetic properties and biocompatibility, but also to the capability of synthesizing SPIONs in various shapes and sizes using several techniques. A comprehensive review of SPION fabrication methods is beyond the scope of our discussion, but can be found elsewhere in the literature. ${ }^{4,9,10}$ One of the key factors in the successful application of SPIONs in clinical imaging and diagnostics is the availability of MRI as a non-invasive and powerful system for tracking magnetic NPs.

When the size of magnetic materials reaches below a single magnetic domain (corresponding to a core diameter of $\leq 20 \mathrm{~nm}$ for iron oxide) superparamagnetism occurs. ${ }^{9}$ By contrast with paramagnetic materials, superparamagnetic NPs do not retain any remnant magnetization when the external magnetic field is removed, which means that their magnetization can be switched on and off. Due to their superparamagnetism and their strong effects on T2 relaxation, SPIONs are the preferred NP agents for MRI contrast. ${ }^{11}$ $\mathrm{T} 1$ and T2 MRI relaxation time constants are under the influence of the local microenvironment. Magnetic inhomogeneity is known as a determining microenvironmental factor in MR imaging influencing the T2 time constant. Thus, the high magnetic susceptibility of SPIONs enables them to alter T2, T2*, or both, and as a consequence, shortens the $\mathrm{T} 2$ and $\mathrm{T} 2 *$ relaxation. As a result, the regions in the proximity of SPIONs can be identified by hypointense signals on the MR image and as such, agents with higher relaxivity are preferred. The $\mathrm{T} 2$ relaxivity for iron oxide NPs is usually at least 5-10 fold higher than T1. ${ }^{12}$ Therefore, most iron oxide-based MRI contrast agents are detected as a signal loss on T2-weighted MR images. The relaxivity of iron oxide-based MRI contrast agents is largely dictated by physiochemical properties such as size, composition, degree of aggregation and surface coating. ${ }^{13}$ Increasing the diameter of these particles leads to an increase in $\mathrm{T} 2$ relaxivities. ${ }^{14}$ Polymeric coatings are also known to increase the $\mathrm{T} 2$ relaxivity. ${ }^{15}$ However, in engineering NPs for in vivo applications, issues such as uptake by the cells of the reticuloendothelial system (RES) and circulation time should also be considered. For example, unmodified NPs can escape internalization by the RES only if there are below $100 \mathrm{~nm}$ in diameter; otherwise, they need to be modified by e.g. PEGylation. ${ }^{16}$

\section{Tailoring SPIONs with targeting moieties}

SPION-based systems are usually composed of an inorganic core surrounded by a coating, which renders the particle biocompatible and confers stability and/or solubility in aqueous solution. Furthermore, the opsonization and circulation time of NPs can be tuned by modulating their size and surface properties (see Figure 1).

Solid tumors usually have a leaky and damaged tumor vasculature, as well as a poorly developed lymphatic drainage system. NPs are believed to accumulate to a higher extent in this 


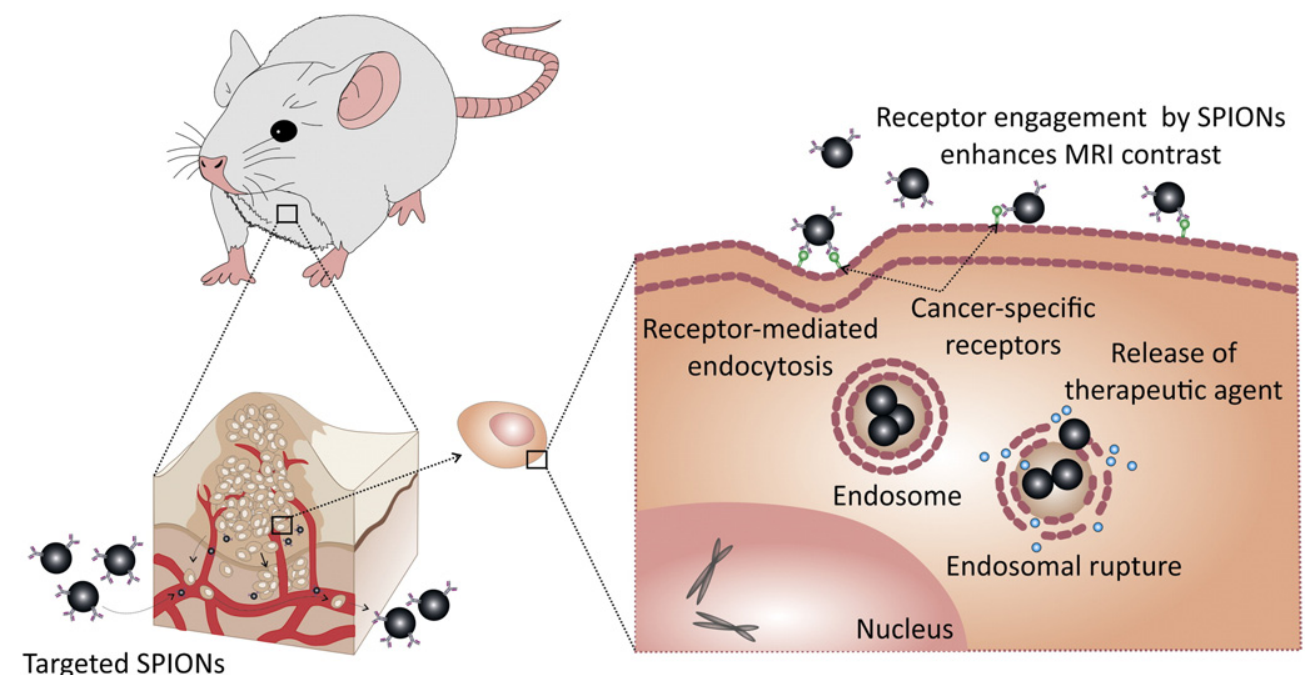

Figure 2. The active targeting scheme for SPIONs and potential application of this strategy in MR imaging and drug delivery.

faulty vascular system than normal tissues, a phenomenon which is called passive targeting using enhanced permeability and retention (EPR effect). ${ }^{17}$ While such targeting strategies have proven to be effective in certain cases - e.g. the uptake of dextran-coated SPIONs was reported to be 10-fold higher in gliosarcoma compared to healthy brain tissue in a rat model ${ }^{18}$ - passive targeting using naked first generation SPIONs usually lacks the required specificity for differentiating specific tumor types from normal tissues.

Therefore, second generation SPIONs are functionalized with targeting moieties, such as monoclonal antibodies (mAbs), aptamers, and small molecule ligands among others, allowing them to recognize specific cellular receptors. Using this scheme, which is called active targeting, ${ }^{19}$ a higher number of NPs can accumulate in the target tissue and enhance the MRI contrast and imaging specificity. For example, targeted imaging is employed for real-time tracking of tumor angiogenesis and growth. ${ }^{20}$ In addition, active targeting limits the off-target effects and minimizes the potential side-effects. An overall scheme for active targeting is depicted in Figure 2.

An alternative targeting strategy that takes advantage of the inherent magnetic properties of magnetic NPs including SPIONs, is known as magnetic targeting. In this strategy, an external magnetic field is applied to the desired location on the body in order to promote the accumulation of injected magnetic NPs in that area. This allows, for example, for targeting cancers close to the human skin. ${ }^{21}$ Passive targeting of SPIONs has recently been reviewed. ${ }^{22}$ In the sections below, active targeting of SPIONs in the diagnosis and detection of cancer will be reviewed.

\section{Common targeting ligands for imaging applications}

Monoclonal antibodies are regarded as the most specific moieties for targeting cancer cells. A list of mAbs and their specific cellular antigens can be found in a review by Allen. ${ }^{23}$ However, there are a number of challenges associated with the application of mAbs as SPION targeting agents. First and foremost is the hydrodynamic size, often up to $20 \mathrm{~nm},{ }^{24}$ which can increase the overall size of the conjugate and as such, reduce NP extravasation and accumulation in the tumor site. ${ }^{25,26}$
Furthermore, with increased size, the NPs' "stealth" characteristics are compromised and more of them are taken up by cells of the RES organs. ${ }^{27}$ Moreover, antibodies can be immunogenic to an extent which cannot be easily predicted, given their complexity and the genetic diversity of the individuals receiving them. ${ }^{28}$ This issue is also very important, since a large number of NPs must be injected for in vivo imaging. These challenges have largely limited the application of mAbs as targeting ligands; however, there are still a number of success stories out there, examples of which will be discussed in the next sections.

To overcome the challenges associated with large antibodies, antibody fragments have been considered as viable functional targeting moieties. Such fragments include, but are not limited to, Fab, ScFv-Fc, ScFv, $\left(\mathrm{Fab}^{\prime}\right)_{2}$, minibodies, diabodies, nanobodies, and peptides. Despite their advantages, fragments have rarely been used for functionalization of SPIONs for imaging applications, ${ }^{29}$ likely due to the complexities associated with their production.

Aptamers are synthetic single-stranded DNA or RNA oligomers used for specific targeting of biomolecules. Nowadays, aptamers are designed through the Systematic Evolution of Ligands by EXponential enrichment (SELEX) method, ${ }^{30}$ a series of sequential in vitro selection, purification and amplification steps for characterization of a specific ligand with high binding affinity for the desired antigen. Other more recent technologies such as cell-SELEX allow for selection of aptamers that can recognize specific cell lines. ${ }^{31}$ Aptamers have important advantages compared to mAbs in that they are much smaller in size and entail an easier manufacturing process. ${ }^{32}$ Moreover, since they are smaller, a large number of distinct aptamers can be accommodated on the surface of NPs, permitting multivalent binding and synergistic affinity (up to 26-fold greater than the intrinsic affinity of individual aptamers). ${ }^{33}$ Aptamers with very high specificity have been engineered, with the ability to even differentiate subtypes of non-small cell lung cancer. ${ }^{34}$ In one such SELEX study after 15 rounds of enrichment, Li and colleagues $^{35}$ selected high affinity DNA aptamers that specifically bind to MDA-MB-231 breast cancer cells. Furthermore, among this panel of aptamers (having a $K_{\mathrm{d}}$, dissociation constant, 

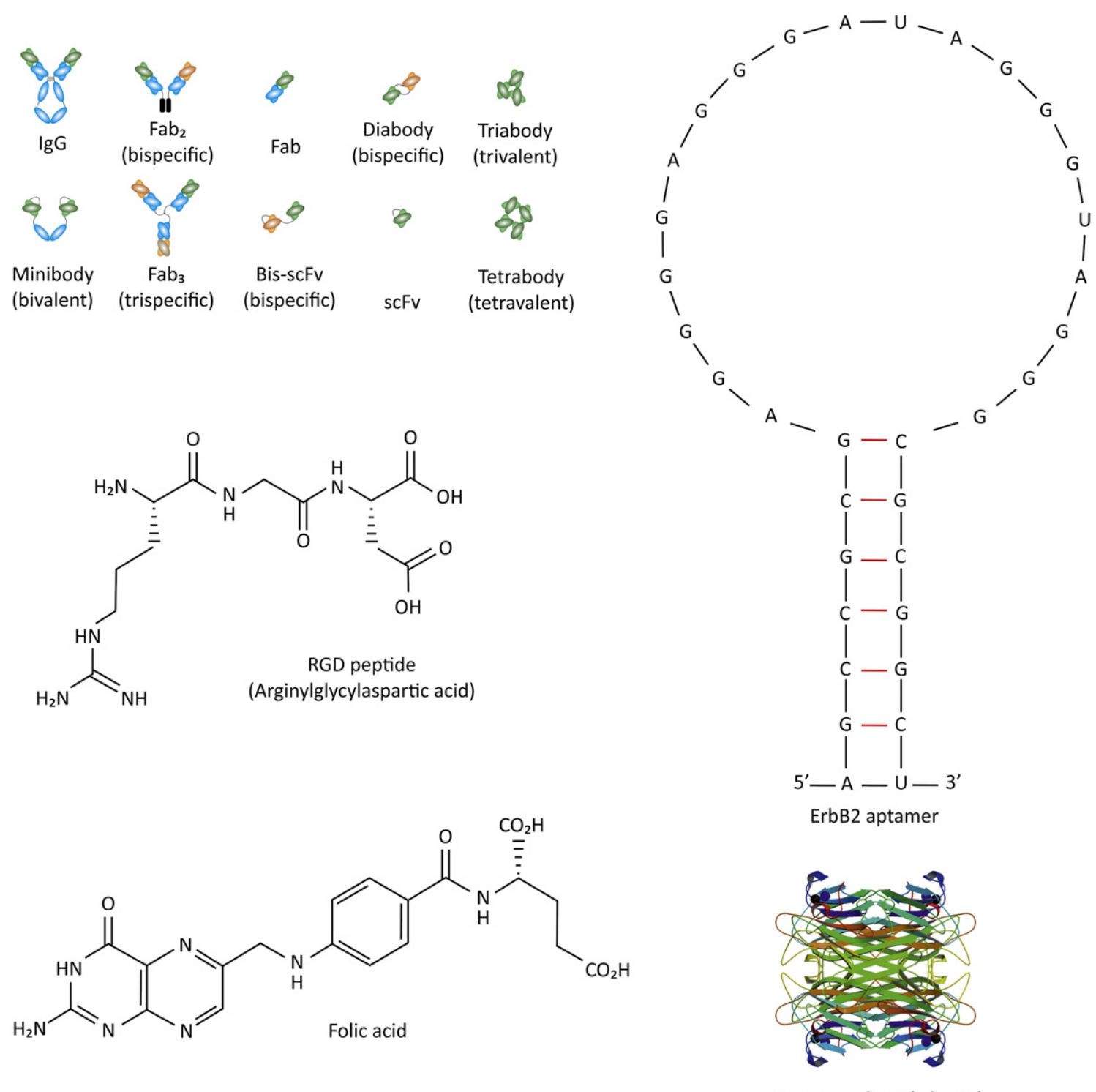

Concanavalin A (a lectin)

Figure 3. Versatile targeting ligands used in SPION-based imaging systems for specific detection of tumor cells. The ErbB2 aptamer sequence has been adapted from Kim and Jeong. ${ }^{36}$ Concanavalin A structure has been derived from RCSB-PDB ID: 2UU8. Reproduced with permission. ${ }^{180}$

of 3-108 nM), they showed that the selected aptamer, LXL-1, exhibited a low $K_{\mathrm{d}}$ value $(44 \pm 8 \mathrm{nM})$ as well as outstanding selectivity for MDA-MB-231 cell recognition. The truncated version of the LXL-1 aptamer, LXL-1-A could distinguish the breast cancer tissue against breast cancer tissue with metastasis in $76 \%$ of the cases. Similar studies of this type have been conducted for selecting other cancer cell-specific aptamers - e.g. against the extracellular domain of ErbB2 ${ }^{36}$ and hnRNP A $1^{37}$ for breast cancer, as well as against epithelial cell adhesion molecule $(\mathrm{EpCAM})^{38}$ and integrins ${ }^{39}$ present in most solid tumors.

Lectins are another class of targeting ligands, which bind to the glycoprotein and glycolipid moieties of glycans on the cell membrane. Since glycans are central to a variety of cellular activities, such as cell-cell communication, adhesion, differentiation and development, their overexpression or aberrant expression on the cell membrane is usually associated with cancer, either as a cause or consequence. ${ }^{40}$ As such, lectins, such as wheat germ agglutinin (WGA), may be employed for targeting of NPs for cancer imaging or therapy. Despite the extensive applications of lectin-NP conjugates in investigating the glycanNP interactions, ${ }^{41}$ there is only a small number of studies using lectin-functionalized NPs for imaging applications.

Cell-penetrating peptides are yet another class of targeting moieties. The trans-activating transcriptional activator (TAT), the first member of this class of molecules, was initially discovered in human immunodeficiency virus 1 (HIV-1). ${ }^{42}$ This peptide sequence was shown to be internalized by a panel of cell lines. Ever since, these peptides have been employed for delivering various molecular cargos into cells, usually via endocytosis. Since then, several cell-penetrating peptides (including RGD) have been discovered and extensively exploited for targeted therapy and imaging. However, they usually lack specificity toward a specific cell type. 
Table 1

A cursory comparison of different targeting moieties.

\begin{tabular}{|c|c|c|c|c|}
\hline Targeting moiety & Specificity & Size & Immunogenicity & Production cost and complexity \\
\hline Monoclonal antibodies & Highly specific & 150 kDa (monomeric IgG) & Possible & Very high \\
\hline Antibody fragments and fusions & Highly specific & $\begin{array}{l}25-140 \mathrm{kDa} \\
\text { (based on fragment structure) }\end{array}$ & Possible & High-very high \\
\hline Lectins & Moderately specific & $\begin{array}{l}36 \mathrm{kDa} \text { (WGA dimer) and } \\
104-112 \mathrm{kDa} \text { (Concanavalin } \mathrm{A})\end{array}$ & Possible & Very high \\
\hline
\end{tabular}
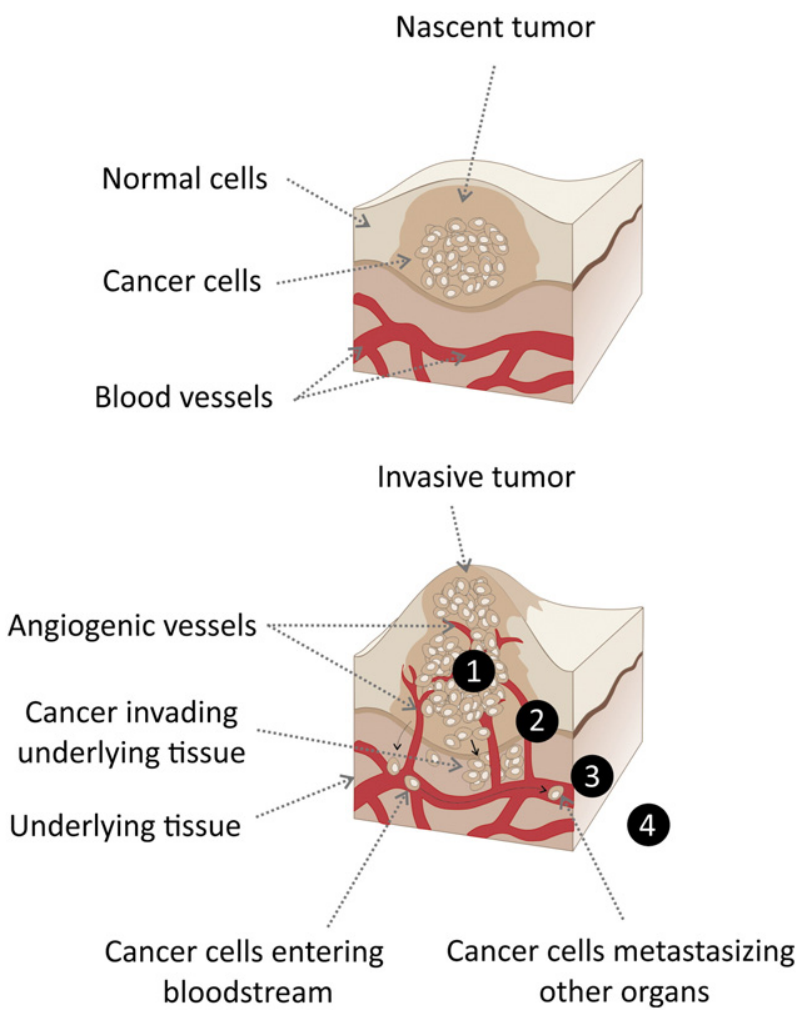

Figure 4. SPIONs can help detect cancer in 4 different stages: 1) detection of receptors overexpressed on cancer cell surface in the tumor mass; 2) detection of unusual angiogenesis in the tumor microenvironment; 3) detection of circulating tumor cells and 4) detection of soluble tumor biomarkers.

Last but not least, small-molecule ligands, such as folic acid, can also serve as SPION targeting ligands. The folate receptor (FR) is a cell-surface receptor which functions in binding and internalizing folate. Since folic acid is pivotal to cancer cell proliferation, it is generally overexpressed in cancer cells ${ }^{43}$; however, it can also be found in minute quantities on the apical surfaces of epithelial cells in a number of normal tissues such as kidney, thyroid, lung, and brain. ${ }^{44}$ This ligand is the one most extensively used for drug delivery and imaging applications. Another small-molecule ligand that is commonly used is methotrexate (MTX). MTX is a chemotherapeutic drug which specifically kills cancer cells overexpressing FR. A scheme representing all targeting moieties discussed herein is provided in Figure 3 and the overall comparison of different targeting moieties with regard to specificity, size, immunogenicity and costs of production has been presented in Table 1 .

\section{Cancer detection}

Several non-targeted SPION-based contrast agents are already available in the clinic for general imaging applications. However, non-targeted SPIONs cannot efficiently accumulate in the tumor site. Targeted delivery of SPIONs can potentially facilitate their accumulation in the vicinity of specific cancer cells and thus improve the sensitivity of MRI. Fabrication and development of SPIONs for detection of specific cancer types and metastases are feasible given the current rate of progress in the field. Next-generation SPION-based MRI contrast agents have been developed by coating the SPION core with suitable materials and conjugating with tumor-specific targeting moieties. These advances have facilitated the use of MRI for early detection of cancer formation and monitoring of treatment efficacy. Overall, SPIONs have been employed for cancer detection using four general strategies: 1) detecting receptors overexpressed on cancer cell surface in the tumor mass; 2) detecting abnormal angiogenesis in the tumor microenvironment; 3) detecting circulating tumor cells and 4) detecting soluble tumor biomarkers (see Figure 4). In the sections below, the progress in the development of targeted SPION-based MRI contrast agents will be reviewed for major cancer types.

\section{Liver cancer}

The first clinical application of SPIONs was in imaging of liver tumors. ${ }^{45}$ This detection strategy is based on the fact that hepatic macrophages, or Kupffer cells, can readily internalize SPIONs in normal hepatic tissue, while liver tumors are mostly devoid of such macrophages. This distinction in macrophagespecific uptake of SPIONs leads to negative enhancement on T2/ T2*-weighted MRI sequences in tumor lesions, facilitating detection of liver tumors or metastases as small as $2-3 \mathrm{~mm}$. The first such SPIONs clinically used in Europe were coated with dextran T10. Later, other SPIONs with different sizes and coatings were introduced into the market. However, none of these early products were targeted. More recent investigations have been centered on targeted SPION products. 

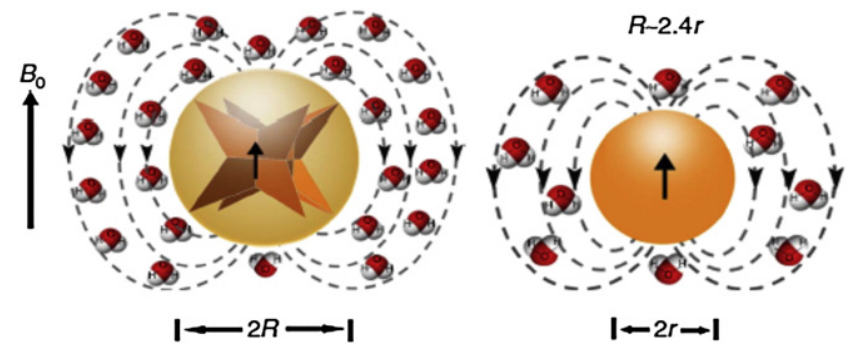

Figure 5. The octapod iron oxide NPs have larger effective valium compared to spherical model with the same geometry. Reproduced with permission. ${ }^{48}$

The previous studies demonstrated that the tuning of size and magnetic strength of iron oxide NPs can be as the cost effective approach to improve the MR imaging. ${ }^{46,47}$ For example, the saturation magnetization and T2 relaxivity of iron oxide NPs enhanced after increasing the NP size. It is easier and more cost effective to tune the physicochemical properties of NPs than NP functionalization with specific targeting ligands. Recently, Zhao et $\mathrm{al}^{48}$ demonstrated that the morphology of iron oxide NPs affects the MR imaging efficacy. They revealed that the octapod iron oxide NPs show higher contrast in T2 imaging of liver cancer compared to spherical model (with the same geometry). The octapod NPs have higher effective volume compared to spherical NPs when they are exposed to external magnetic field (Figure 5). They were also shown to induce more inhomogeneous local magnetic field which in turn leads to contrast enhancement. Indeed, the excellent potency of octapod NPs in MR imaging is related to their unique morphology.

Other studies have focused on engineering targeted SPIONs. Anti- $\alpha$-fetoprotein and anti-glypican 3 antibodies can be used for targeting of hepatocellular carcinoma. The carboxylated dextran-coated ultrasmall SPIONs (USPIONs) conjugated with each one of these antibodies were shown to perform as well as bare USPIONs in shortening T1 and T2 relaxation. ${ }^{49}$ Transmission electron microscopy (TEM) images demonstrated the specific internalization of anti-glypican 3-USPION and anti- $\alpha$-fetoprotein-USPION probes into HepG2 through the GPC3 or AFP receptors, while none or only a few non-targeted USPIONs gained entry into the cell cytoplasm.

Maeng and coworkers ${ }^{50}$ developed a polymeric NP, composed of poly (ethylene oxide)-trimellitic anhydride chloride-folate, doxorubicin (DOX), and SPIONs for dual imaging and delivery. The polymeric NPs were competitively internalized in FR-expressing Hep3B and KB cells, which was shown by reduction in NP uptake when cells were co-treated with FA. The presence of stained vesicles within the tumor cells indicated that NPs were internalized through FR-mediated endocytosis. Subsequently, the efficacy of DOX-carrying NPs was tested in rat and rabbit liver cancer models, and compared with free DOX and a commercial liposomal DOX formulation, DOXIL ${ }^{\circledR}$. The new formulation was selective for FR-expressing tumors and decreased the tumor volume two- and four-fold more than free DOX and DOXIL ${ }^{\circledR}$, respectively. Moreover, immunohistochemical analyses showed that the expression of CD34 (a marker of angiogenesis) and Ki-67 (a marker of cell proliferation) was lower in the group treated with the folate-conjugated polymeric
NPs, and many apoptotic cells were shown to be present, as assessed by the terminal deoxynucleotidyl transferase dUTP nick end labeling (TUNEL) assay. Furthermore, in comparison with Resovist $^{\circledR}$, a commercially available MRI contrast agent, this folate-conjugated polymeric NP showed higher MRI sensitivity, even with its lower iron content $(2.5 \mathrm{mg} / \mathrm{mL}$ vs. $27.9 \mathrm{mg} / \mathrm{mL})$. At the same Fe concentration, the MRI contrast enhancement was higher with the folate-conjugated polymeric NPs than with Resovist ${ }^{\circledR}$, both in vitro and in vivo.

EpCAM has also been used as a marker for targeted selection and detection of hepatocellular carcinoma cells. In a study by Pilapong and coworkers, ${ }^{51}$ aminosilane-coated iron oxide NPs were conjugated with DNA-based EpCAM aptamers (hydrodynamic size $<100 \mathrm{~nm}$ ). TEM studies demonstrated that EpCAMconjugated NPs were highly internalized into HepG2 cells through receptor-mediated endocytosis. The majority (96\%) of SPION-bearing EpCAM-positive HepG2 cells could be isolated with magnetic-activated cell sorting (MACS) and could be successfully detected using MR phantom imaging in vitro.

\section{Prostate cancer}

Similar to some other types of solid tumors, the cure rate of prostate cancer is highly dependent on how early the disease is detected. For several years, Cambidex, non-targeted ferumoxtran10 coated with dextran T10, was considered a success story as a contrast agent for detection of prostate cancer metastases. This USPION-based product was intended for use in differentiating metastatic from non-metastatic lymph nodes in patients diagnosed with prostate cancer with suspected lymph node involvement. However, this experimental product failed to gain FDA approval and its production was discontinued. ${ }^{52}$

Current research is focused on specific targeting of prostate cancer cells. Prostate-specific membrane antigen (PSMA), a $100 \mathrm{kDa}$ cell-surface glycoprotein, is a marker for prostate cancer cells, and has relatively low expression in normal prostate as well as in kidney, brain, and small intestine. ${ }^{53}$ PSMA is also expressed by the vascular endothelium of all solid sarcomas and carcinomas. ${ }^{54}$ The intracellular domain of PSMA is masked by the cell membrane and remains invisible to anti-PSMA antibodies in blood; therefore, binding agents against PSMA should target its extracellular domain. ${ }^{55,56}$ Based on this fact, Abdolahi et al conjugated SPIONs with J591 mAb, which specifically recognizes and binds to the extracellular domain of PSMA. ${ }^{57}$ J591 is among the most promising candidates for targeting viable prostate cancer cells for both detection and treatment. ${ }^{58}$ The purpose of their study was to enhance the delivery of contrast agent to PSMA-expressing cells, thereby enhancing contrast in those cells. The authors also fabricated probes for PSMA-expressing lymph node carcinoma of the prostate (LNCaP) cells, and demonstrated their high specificity for these cells as compared with PSMA-negative DU145 cells.

Wang et $\mathrm{al}^{59}$ have developed a PEGylated, thermally cross-linked SPION (TCL-SPION) that provides MRI contrast combined with simultaneous DOX delivery to cancer cells. TCL-SPIONs were functionalized with A10 aptamer, which binds to the extracellular domain of PSMA, for targeting prostate cancer (Figure 6). The differential internalization of aptamer- 

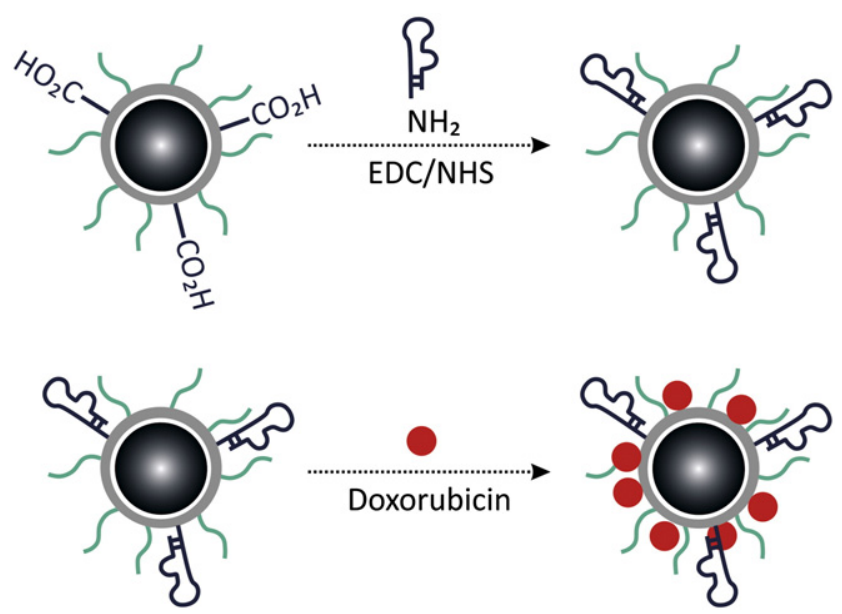

Figure 6. TCL-SPION-A10 aptamer bioconjugate system with intercalated DOX molecules. Redrawn with permission. ${ }^{59}$

conjugated TCL-SPIONs in PSMA-expressing cells was studied by comparing the uptake of non-targeted and targeted TCL-SPIONs in PSMA-expressing LNCaP cells and non-PSMA-expressing PC3 cells. Only the targeted TCL-SPIONs were internalized in LNCaP cells $3 \mathrm{~h}$ after incubation and their accumulation increased with time. Neither of the NPs could be internalized in PC3 cells. The change in $\mathrm{T} 1$ and $\mathrm{T} 2$ relaxation times in $\mathrm{LNCaP}$ cells was not prominent upon incubation with non-targeted TCL-SPIONs, while the targeted SPIONs dramatically decreased T1 and T2 (T1: $1939 \pm 116$ to $263 \pm 23 \mathrm{~ms}$; T2: $104.2 \pm 1.4$ to $26.6 \pm 0.4 \mathrm{~ms})$. Furthermore, as expected, the targeted SPIONs could not significantly decrease the $\mathrm{T} 1$ and $\mathrm{T} 2$ in non-PSMA expressing PC 3 cell lines. In a very similar study, ${ }^{60}$ another group conjugated quantum dots onto the surface of a nanocomposite composed of a $150 \mathrm{~nm}$ spherical polystyrene matrix and embedded with $10 \mathrm{~nm}$ SPIONs. Furthermore, the chemotherapeutic agent paclitaxel was loaded onto the surfaces of the composites using a PLGA layer and the construct was further functionalized with anti-PSMA antibody for specific detection of LNCaP cells. The inclusion of quantum dots and SPIONs in the same system makes it possible to perform bimodal imaging by fluorescence and magnetic resonance, respectively.

Ghosh and coworkers ${ }^{61}$ developed new generation of carriers which deliver large number of contrast agents per each cell receptor. They arranged magnetic NPs on the M13 filamentous virus which displayed a targeting peptide that specifically binds to the SPARC glycoproteins. The magnetic NP-M13 showed higher contrast efficacy in imaging of prostate cancer compared to SPIONs directly bound to the targeting peptide (Figure 7). The functionalization of carriers with targeting ligands can be a promising approach to improve the targeted delivery of contrast agents per cell. Using this strategy, the challenges of cell receptor number/accessibility and overloading of targeting ligands were tackled. This approach provides unique opportunities for targeted imaging of different tumor cells, since it is possible to display the desired targeting peptide using genetically engineered M13.

Prostate stem cell antigen (PSCA) is another prostate tumor biomarker target. PSCA expression is associated with tumor stage and androgen-independence. As such, Gao et $\mathrm{al}^{62}$ synthesized multifunctional NPs composed of a poly(D,L-lactic-co-glycolic acid) (PLGA), docetaxel, and SPIONs. To this core, a multilayer poly(allylamine hydrochloride) shell was added, and then the construct was further conjugated with two different-sized PEG molecules, and a single-chain PSCA antibody. In vitro, the NPs could sustain drug release over $764 \mathrm{~h}$ and caused significant enhancement in MRI contrast in PC3M cells. Targeting with the antibody enhanced NP internalization by 1.5 -fold compared to non-targeted NPs in PC3M cells after $2 \mathrm{~h}$ of incubation. Furthermore, the targeted NPs specifically enhanced MRI contrast in vivo in male nude mice bearing PC $3 \mathrm{M}$ xenografts. When used to treat mice, the NPs reversed tumor growth and significantly increased the life span of mice. Since docetaxel is the main chemotherapeutic for treatment of hormone-refractory prostate cancer, these NPs have important implications in imaging and drug delivery for this disease.

\section{Brain tumors}

The biodistribution and targeting efficacy of NPs are mainly governed by the biological barriers. The NPs can easily obtain access to the cancer cells/tissues directly exposed to the blood stream while their journey to the pre-angiogenic and brain tumors having no direct link to the blood is very difficult. ${ }^{63}$ Due to blood brain barrier (BBB), there are many challenges associated with the early detection and treatment of brain tumor. ${ }^{64}$ In order to detect the pre-angiogenic and brain tumors at the early stages, it is required to fabricate NPs capable of penetrating the defense barriers. Cao et al, ${ }^{65}$ synthesized magnetoferritin NPs capable of transport through the endothelium, epithelium and BBB via clathrin-mediated endocytosis and specifically detected the tumor cells which expressed the transferrin receptor 1 . Indeed, the magnetoferritin NPs can be a promising candidate to overcome the challenges associated with the NP biodistribution and targeted imaging of brain cancer.

Brain tumors are perhaps the most attractive targets for imaging applications using USPIONs. ${ }^{66}$ In this regard, the efficacy of non-targeted Ferumoxtran-10 and its derivative ferumoxytol contrast agents has been already shown in MRI, e.g. in central nervous system (CNS) lesions such as lymphoma, multiple sclerosis (MS), acute disseminated encephalomyelitis, and vascular lesions. ${ }^{67-69}$ SPIONs can be conjugated with moieties that are specific for various CNS targets, including gliomas. Gliomas are among the most common brain tumors. ${ }^{70}$ The surgical resection of these tumors largely depends on delineating the tumor margins using an effective imaging modality. ${ }^{71}$ For example, the glioma-specific chloride ion channel is sensitive to chlorotoxin, a modified small peptide isolated from scorpion Leiurus quinquestriatus venom. ${ }^{72}$ Chlorotoxin has been employed for glioma targeting in a severe combined immunodeficient (SCID) mouse model bearing xenograft gliomas. ${ }^{73}$ In a study by Meng et $\mathrm{al}^{74}$ SPIONs were conjugated with chlorotoxin and the fluorescent dye, fluorescein isothiocyanate (FITC), such that the targeting of human U251-MG and rat C6 glioma cells could be confirmed by MRI and the amount of NP uptake could be measured by inductively-coupled plasma emission spectroscopy. Confocal laser scanning microscopy was used for optical imaging of functionalized NPs. The conjugation of chlorotoxin to the NPs significantly enhanced preferential SPION 


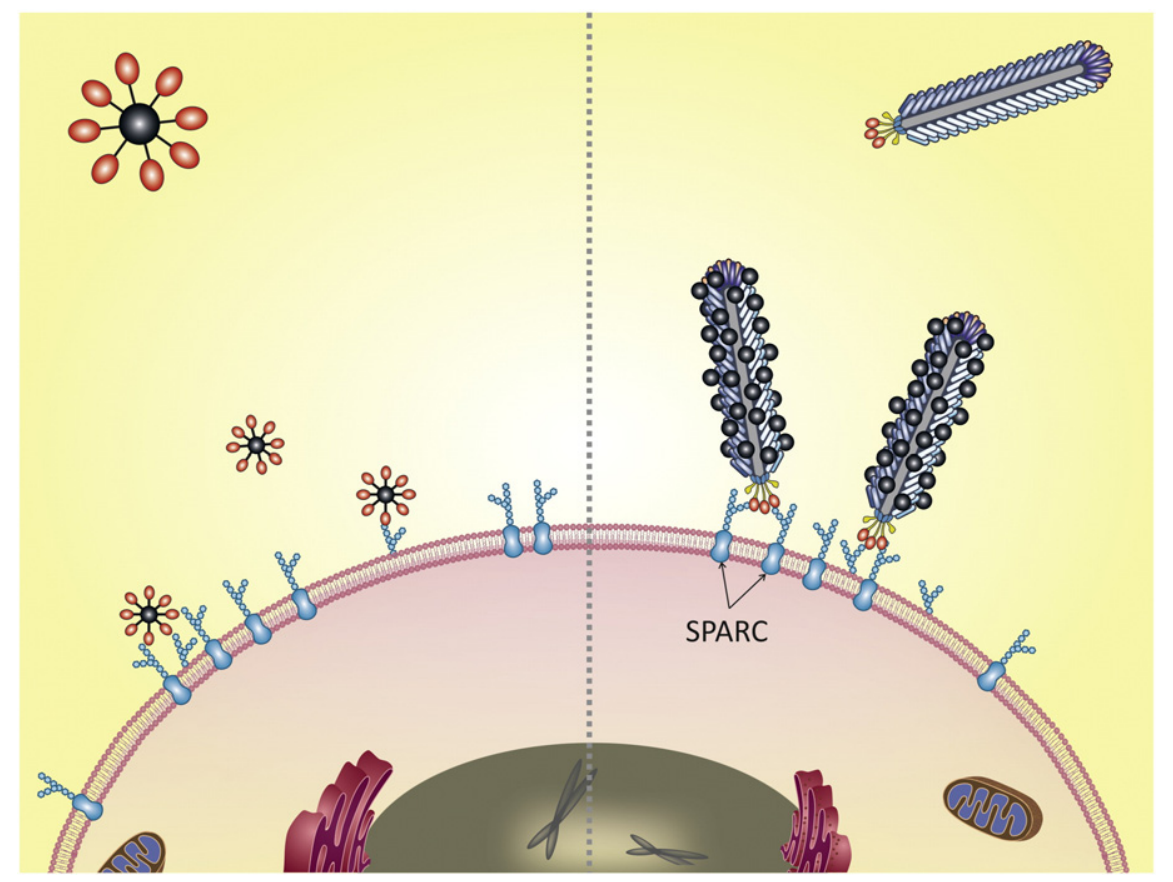

Figure 7. Left panel: tumor targeting with the NPs directly bound to the ligands; right panel: tumor targeting with large number of NPs arrayed on the genetically engineered M13 displaying SPARC binding peptides. Redrawn with permission. ${ }^{61}$

uptake, demonstrating specific targeting of glioma cells. A similar study also demonstrated that PEG-coated iron oxide NPs conjugated with chlorotoxin can function as glioma-specific MRI contrast agents. ${ }^{75}$ The NPs were coated with PEG to prolong serum half-life in vivo by reducing protein adsorption and blocking non-specific uptake by RES macrophages. Furthermore, the terminal functional groups of PEG could be utilized for conjugation of ligands (e.g., chlorotoxin) and other potential therapeutic agents. The favored NP localization in gliomas increased the MRI contrast in 9L cells and athymic (nu/nu) mice bearing 9L gliosarcoma xenografts. The internalization of NPs into the cytoplasm of 9L cells was validated using TEM.

Shevtsov et $\mathrm{al}^{76}$ conjugated SPIONs with heat shock protein Hsp70 for glioma imaging. The Hsp70-SPIONs enhanced the MRI contrast in an orthotopic model of 9L glioma compared with the non-targeted NPs. This high extent of contrast enhancement was due to the accumulation of Hsp70-SPIONs in the gliomas, as shown by histological analysis. There was a 40 -fold greater uptake of Hsp70-SPIONs in tumor than in normal brain tissue.

Transferrin receptor (TfR) is a transmembrane glycoprotein involved in the uptake of the iron-chelating protein, transferrin (Tf). Since iron is a fundamental element that plays major roles in metabolism, DNA replication, and proliferation, highly proliferating cells, such as cancer cells, usually have high iron demands. Therefore, though TfR is expressed in normal tissues, it is usually overexpressed in cancer cells. ${ }^{77}$ Since $\mathrm{Tf}$ is internalized through a receptor-mediated pathway, it has been extensively used as a targeting ligand in both imaging and drug delivery systems. Furthermore, given the fact that the $\mathrm{Tf}$ internalization cycle takes only 5 minutes to complete - corresponding to 20,000 Tf molecules/ cell $/ \mathrm{min}^{78}$ - a large number of Tf-targeted SPIONs can be internalized. SPIONs have been exploited for cell-sorting in both biomedical and industrial applications. For example, Durgadas et $\mathrm{al}^{79}$ fabricated fluorescent superparamagnetic quantum clusters by fusing near-infrared (NIR) fluorescent bovine serum albumin (BSA)-stabilized gold clusters with dopamine-anchored SPIONs. Subsequent addition of $\mathrm{Tf}$ to the resulting clusters lent targeting capabilities to the hybrid clusters, as well as increased hemocompatibility. The fabricated NPs could selectively eliminate C6 glioma cells from blood and urine samples. Fluorescence could be detected at the single-particle level, demonstrating the exquisite sensitivity of the system. Therefore, circulating tumor cells could be detected in a multimodal manner.

Jiang et $\mathrm{al}^{80}$ conjugated $\mathrm{pH} /$ temperature-sensitive magnetic poly $(N$-isopropylacrylamide-co-acrylic acid) nanogels with Cy5.5-labeled lactoferrin (hydrodynamic size of $95.5 \pm 6.2 \mathrm{~nm}$ ), which is known to target glioma. ${ }^{81}$ Under physiological conditions in blood and normal tissues, the nanogels were hydrophilic and swollen, which increased their blood circulation time. In the lower $\mathrm{pH}$ environment of tumor tissues ( $\mathrm{pH} 6.8$ vs. 7.4 in blood), the nanogels became hydrophobic and shrank, which enhanced their internalization by tumor cells (see Figure 8). These multifunctional nanogels enabled in vivo MRI and ex vivo fluorescence imaging with high sensitivity and specificity in a C6 glioma rat model. $6 \mathrm{~h}$ post-injection, the targeted magnetic nanogels generated a dramatic contrast enhancement in the tumor. There was a gradual decrease in MR signal of $47-82 \%$ in individual rats at $48 \mathrm{~h}$ post-injection.

Another protein ligand for imaging of brain tumors is endothelial growth factor (EGF). In one study, ${ }^{82}$ SPIONs were conjugated with recombinant human EGF. The targeted NPs showed high internalization and no toxicity against $\mathrm{C} 6$ cells. An orthotopic model of C6 gliomas was used to investigate NP accumulation and biodistribution in tumors by MR imaging. NPs 
A

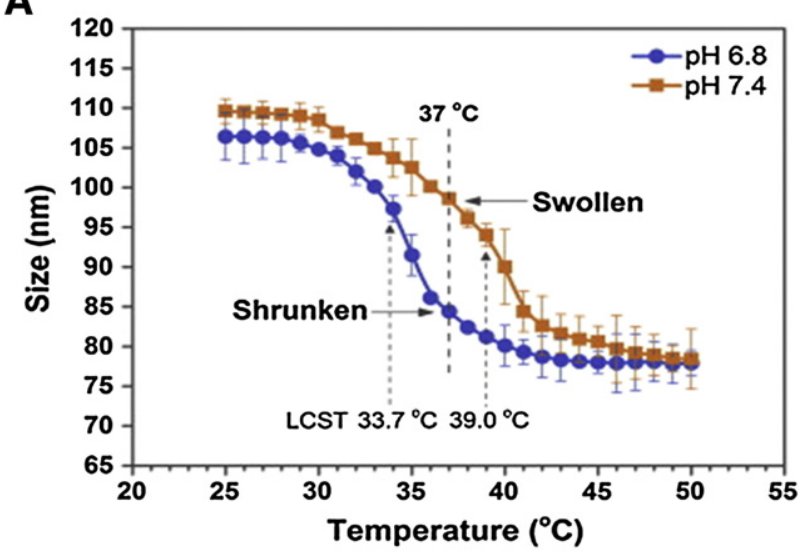

B

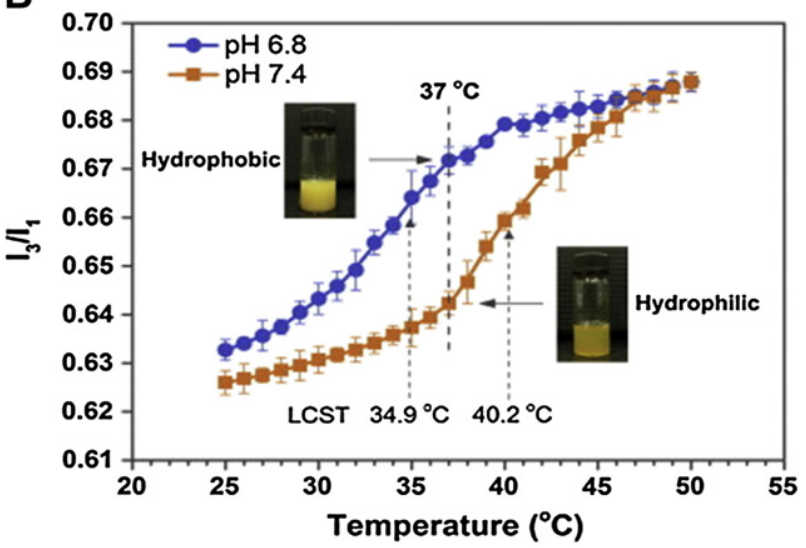

Figure 8. The stimuli-response curves of magnetic nanogels. (A) Size change of the nanogel in response to temperature at $\mathrm{pH} 6.8$ and 7.4. (B) The hydrophilicity/hydrophobicity of the nanogels in response to temperature at pH 6.8 and 7.4. Reproduced with permission. ${ }^{80}$

produced significant negative contrast in tumors, which could be seen as hypo-intense regions on T2-weighted images.

Small-molecule ligands such as MTX have also been utilized in targeting gliomas. For example, in one study, ${ }^{83}$ iron oxide NPs modified with a PEG self-assembled monolayer (SAM) were functionalized with MTX for simultaneous drug delivery and MR imaging. The internalization of targeted NPs was significantly higher than their non-targeted counterparts in 9L cells. The NPs provided significant contrast enhancement in cultured 9L cells and were more cytotoxic than free MTX. The MTX antidote, leucovorin, reversed the cytotoxicity of the conjugates and free MTX, showing that the observed cytotoxicity is due to the bound MTX and not the conjugate. Since the conjugates retained their crystal structure up to $144 \mathrm{~h}$ after internalization, as shown by electron diffraction analysis, they can be used for long-term imaging of tumor cells over the course of treatment.

\section{Breast cancer}

Several breast cancer cell markers such as FR, Her2/neu receptor, Tf receptor (TfR), ErbB and integrins have been used for targeting of SPIONs to breast cancer tissue for imaging and diagnostic applications.

In a study by Sun et al, ${ }^{84}$ SPIONs were initially coated with covalently-bound bifunctional PEG and subsequently conjugated with folic acid. The NPs were shown to preferentially target the FR-overexpressing HeLa cells and not the non-FR expressing MG-63 osteosarcoma cells. The uptake of folic acid-functionalized NPs in HeLa cells was 12-fold higher than non-targeted NPs after $4 \mathrm{~h}$ of incubation. The functionalized NPs caused a significant contrast enhancement in $\mathrm{HeLa}$ cells compared to MG-63 cells in vitro. Furthermore, a competitive inhibition assay in the presence of free folic acid demonstrated the specificity of the nanoconjugate.

Kohler et al ${ }^{85}$ have developed iron oxide NPs modified with a self-assembled monolayer (SAM) of (3-aminopropyl)trimethoxysilane and covalently bound with MTX, which can be used for simultaneous MR imaging and drug delivery to breast cancer. The SAM-modified NPs were conjugated with MTX through an amide bond, such that MTX could be cleaved from the NPs in the low $\mathrm{pH}$ environment of lysosomes after cellular uptake. In human MCF-7 and HeLa cancer cell lines, MTX release was monitored by UV absorbance (at $304 \mathrm{~nm}$ ) under conditions that simulated lysosomal $\mathrm{pH}$ and protease levels. Finally, cells expressing the FR were shown to internalize the NP to a higher extent than non-FR expressing cells.

Leuschner et $\mathrm{al}^{86}$ have shown that the specificity of luteinizing hormone releasing hormone (LHRH) and chorionic gonadotropin (CG)-bound SPIONs in human breast cancer cells depends on the level of receptor expression in target cells as well as the length of incubation. The neutral charge of these NPs improves their cellular uptake and helps them to avoid uptake by RES macrophages. The internalization of LHRH-conjugated SPIONs was significantly higher than CG-conjugated SPIONs. Furthermore, the accumulation of LHRH-conjugated SPIONs was approximately 9 fold higher than that of non-targeted SPIONs inside human breast cancer MDA-MB-435S.luc cells. Ligand specificity was demonstrated by a decrease in intracellular $\mathrm{Fe}$ content, as measured by Prussian blue staining, upon co-incubation of the SPION conjugates with free ligand. Another advantage of LHRH-conjugated SPIONs was poor incorporation within macrophages. LHRH-conjugated SPIONs were mainly localized in human breast cancer xenografts in a nude mouse model, subcutaneously injected with $10^{6}$ MDA-MB-435S.luc cells in the interscapular region. On the other hand, non-targeted SPIONs were mostly detected in the liver and not in the tumor. In addition, the concentration of LHRH-conjugated SPIONs in the lungs directly corresponded to the number of metastatic cells present (77.8 pg Fe/metastatic cell), while non-targeted SPIONs did not accumulate in the metastatic lesions of the lung in mice. As such, these MRI contrast agents have potential applications in detection of metastases in lymph nodes, bones, and peripheral organs.

Her2/Neu receptors are among the most specific markers in targeting, staging, and treatment of breast cancer. Chen et al ${ }^{87}$ have fabricated SPIONs functionalized with dextran and Herceptin. The dextran coating improved NP dispersibility in solutions of various $\mathrm{pH}$ ranges and enhanced their saturation magnetization (up to $80 \mathrm{emu} / \mathrm{g}$ ). Moreover, the synthesized NPs were minimally cytotoxic against a variety of cell lines. MR 


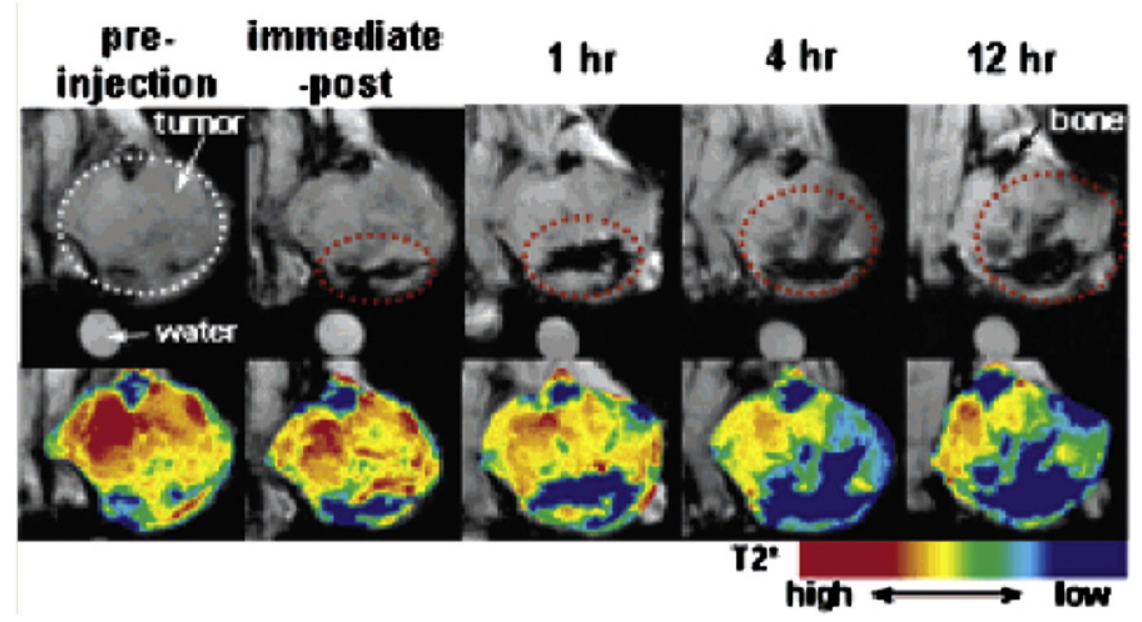

Figure 9. T2*-weighted MR images of a mouse implanted with NIH3T6.7 cancer cells at $9.4 \mathrm{~T}$ along with their color maps at different time points after NP injection. Tumor area has been shown with white dotted lines. Immediately after injection, a hypointense area (red dotted circle in the figure) appears at the bottom of the tumor, which subsequently diffuses to the central and upper region of the tumor in a gradual manner. When the same MR images are subjected to color mapping, more details of the MR signal changes are revealed, such that the progressive diffusion and targeting events of the NPs into the tumor tissue can be seen as blue and green colors, corresponding to occupation of roughly $1 / 3,1 / 2$, and $2 / 3$ of tumor tissues at 1,4 , and $12 \mathrm{~h}$, respectively. Reproduced with permission. ${ }^{89}$

enhancement among the 4 tested breast cancer cell lines, BT-474, SKBR-3, MDA-MB-231, and MCF-7, was greater in cell lines with higher HER2/neu expression, validating the targeting specificity. However, even cell lines with low HER2/ neu expression levels could be adequately detected. Upon intravenous injection of these NPs into mice bearing breast tumor allografts, a $45 \%$ drop in MR enhancement was seen in T2-weighted MR images, which indicated the high accumulation of NPs within the tumor site. A proportional enhancement in T2 MR images with respect to the number of receptors in different cell lines has also been observed with streptavidin-conjugated SPIONs functionalized with biotinylated mAb. ${ }^{88}$ In a similar study, Huh and coworkers ${ }^{89}$ synthesized magnetic iron oxide nanocrystals conjugated to Herceptin, which can selectively target human cancer cells implanted in live mice in vivo. These nanocrystal probes were further conjugated with fluorescent dye-labeled antibodies, allowing them to be used both for in vitro and ex vivo optical detection of cancer as well as for in vivo MRI. Most importantly, this system allowed for detailed monitoring of progressive cancer targeting at high magnetic fields $(9.4 \mathrm{~T})$, revealing important information about the complex vascular structures of the tumor tissue (Figure 9).

The effective treatment of stage 4 breast cancer relies on the detection of small metastases. Kievit et al ${ }^{90}$ coated $8 \mathrm{~nm}$ SPIONs with a chitosan/PEG copolymer, labeled them with a fluorescent dye (Alexa Fluor 647 for in vivo or Oregon Green 488 for in vitro detection), and further functionalized them with a mAb against the Neu receptor (final hydrodynamic size of 40-47 nm). As a negative control, SPIONs were conjugated with mouse IgG. The Neu-functionalized NPs were highly internalized by Neu-expressing mouse mammary carcinoma (MMC) cells, and specificity to the Neu antigen was demonstrated by reversal of the internalization in the presence of free Neu antibody. Not only could the Neu-functionalized NPs target primary breast cancer tumors, they were also capable of recognizing liver, lung, and bone marrow metastases in the transgenic mouse model of metastatic breast cancer. This binding led to significant contrast enhancement in MR images of both primary tumors and micrometastases. Since non-targeted NPs failed to bind to these metastases, it can be deduced that active targeting is essential for delivery.

Kresse et al $^{91}$ covalently coupled the human Tf to USPIONs for in vivo imaging of SMT/2A tumor-bearing rats (rat mammary carcinoma). With a half-life of $17 \mathrm{~min}$ in normal rats, the conjugates could reduce the MRI signal of the tumor by $40 \%$ (range 25-55\%) $150 \mathrm{~min}$ after injection, and this signal reduction could be maintained for at least $8 \mathrm{~h}$. By comparison, the identical parent USPIONs or those labeled with human serum albumin could only induce a $10 \%$ reduction in tumor signal over time.

Integrins are heterodimeric transmembrane glycoproteins that facilitate cell-matrix adhesion by acting as receptors for molecules of the extracellular matrix (ECM). These interactions underlie the induction of many signaling pathways that are involved in cell-ECM communications. Among the different combinations of $\alpha$ - and $\beta$-subunits (which give rise to 24 integrin molecules), $\alpha_{v} \beta_{3}$ is of particular interest as an in vivo imaging target, since it is known to be involved in the process of angiogenesis, tumor development, and metastases, and can thus be found on angiogenic vessels in malignant tumors. Indeed, paramagnetic particles conjugated with LM609 mAb, which targets $\alpha_{\mathrm{v}} \beta_{3}$ expression, have been used for in vivo MRI detection of angiogenesis. ${ }^{92}$ The majority of integrins, including $\alpha_{v} \beta_{3}$, show a high binding affinity for arginine-glycine-aspartic acid (RGD) peptides, which naturally occur in ligands specific for integrin receptors. Consequently, RGD peptides have been extensively used as targeting moieties for functionalization of different NP systems, including SPIONs. Usually, due to higher affinity and in vivo stability, the cyclic form of RGD peptides is preferred over their linear counterparts. ${ }^{93}$ Furthermore, unlike other tumor markers, due to the expression of $\alpha_{v} \beta_{3}$ integrin on 
the apical and basolateral sides of proliferating blood vessels in a tumor, RGD-targeted NPs can directly access these receptors from the bloodstream, which reduces the need for NP extravasation into the tumor microenvironment. ${ }^{94}$ In addition, in certain cancer types, $\alpha_{\mathrm{v}} \beta_{3}$ expression correlates with the tumor grade. ${ }^{95}$ Thus, not only can integrins function as markers for tumor imaging, but they also provide additional prognostic information. SPIONs have also been conjugated with cRGD, targeting integrins in a BT-20 breast tumor model. ${ }^{96}$ In this study, the level of $\alpha_{\mathrm{v}} \beta_{3}$ integrin expression, NP pharmacokinetics (mainly half-life), and tumor vascularization density (related to the rate of NP escape from the vasculature) dictated NP targeting efficiency. Due to uptake by integrin-expressing cells, the targeted cRGD-conjugated SPIONs had a shorter plasma half-life than non-targeted NPs (180 vs. $508 \mathrm{~min})$. However, even given their short half-life, the targeted SPIONs could still target integrins on tumor cells. Since these NPs were also functionalized with Cy5.5, $\alpha_{\mathrm{v}} \beta_{3}$ integrin-expressing tumor cells could also be visualized with fluorescence reflectance imaging (FRI) and fluorescence molecular tomography (FMT) in vivo. A similar study has shown the specific targeting of $\alpha_{v} \beta_{3}$-rich glioblastoma and breast cancer, both in vitro and in vivo, using $\mathrm{c}(\mathrm{RGDyK})$-functionalized USPIONs. ${ }^{97}$ In another similar approach to targeting $\alpha_{\mathrm{v}} \beta_{3}$ for breast cancer imaging, Yan and colleagues ${ }^{98}$ prepared PEG-modified and SPIONencapsulated magnetoliposomes with biotin. In a guided 3-step targeting approach, biotinylated anti- $\alpha_{\mathrm{v}} \beta_{3}$ mAbs were intravenously injected into mice bearing MDA-MB-435S breast tumors, followed by injection of avidin/streptavidin and finally, injection of the biotinylated magnetoliposomes. Fluorescence immunohistochemistry and Prussian blue staining were employed to determine the specificity of $\alpha_{\mathrm{v}} \beta_{3}$ targeting. The 3-step pre-targeting approach enhanced the MRI contrast to a larger extent compared with no targeting $(7.0 \%$ of the tumor area vs. $2.0 \%$ ), and the targeted magnetoliposomes were shown to colocalize with neovasculature. In another study, ${ }^{99}$ iron oxide NPs were coated with milk casein protein to generate NPs with the final hydrodynamic size of $\sim 30 \mathrm{~nm}$. Not only were the fabricated NPs water soluble with acceptable colloidal stability and biocompatibility, but they also had a transverse relaxivity $\sim 2.5$-fold higher than that of identical core NPs with an amphiphilic polymer coating. The NPs demonstrated strong contrast enhancement in liver and spleen.

Gong et al ${ }^{100}$ fabricated dual-targeting micelles encapsulating SPIONs from the amphiphilic block copolymer poly(ethylene glycol)-poly( $\varepsilon$-caprolactone) (PEG-b-PCL) conjugated at the distal ends of the PEG block with cRGD and ScFv-ErbB single chain antibody. The efficiency of fabricated contrast agent was assessed on an animal model produced by subcutaneous injection of BT474 cells into the BALB/c-nu female nude mice with a $1.5 \mathrm{~T}$ clinical MRI scanner. The dual-targeted magnetic micelles delivered by tail vein injection decreased the T2 signal intensity of the tumor in animals to a higher extent than singleligand targeted and non-targeted magnetic micelles.

Foy et al, ${ }^{101}$ designed an optical imaging based strategy to assess the biodistribution and localization of magnetic NPs in mice with xenograft breast cancer. To this end, the magnetic NPs were modified by near infrared dyes and the circulation time, passive (in the absence of magnetic field), and active (in the presence of magnetic field) targeting of NPs were tracked by optical imaging. The authors demonstrated that tumor accumulation and cancer detection efficacy of magnetic NPs exposed to magnetic field were considerably higher than those with no external magnetic field. Therefore, the combination of advanced imaging with magnetically guided delivery of NPs may provide exclusive opportunity to detect cancer cells in early stages.

The discovery and characterization of novel breast cancer markers will usher in new targeting moieties. New markers, such as low density lipoprotein receptor-related protein 5 (LRP5), ${ }^{102}$ low-density lipoprotein receptor 6 (LRP6), ${ }^{103}$ and others, may prove to be applicable for imaging of breast cancer and its metastases in the future.

\section{Lung cancer}

In cancer patients, treatment protocols and disease-management strategies are largely based on the presence or absence of metastases. Since lung is the organ most commonly invaded by metastases, ${ }^{104}$ early detection of metastasis can significantly contribute to improving the treatment and/or prognosis of affected patients. Therefore, probes which can differentiate metastatic cancer cells are highly desirable. Lung MRI is challenging due to the low occupancy (20-25\%) of the lung volume by solid tissue (as most of the lung volume is comprised of air). As a result, lung usually generates weak intrinsic MRI signals. Furthermore, due to the presence of many air-tissue interfaces in the lung, substantial field gradients are generated which further weaken the MRI signal (T2* of $1 \mathrm{~ms}$ at $1.5 \mathrm{~T}$ in humans ${ }^{105}$ and $<0.8 \mathrm{~ms}$ at $2 \mathrm{~T}$ for small animals ${ }^{106}$ ). The advent of hyperpolarized (HP) $3 \mathrm{He}$ and 129Xe MRI has paved the way for high resolution imaging of the lung, since the high diffusivity of these gases can negate most of the field gradients generated at the alveolar walls. In this regard, Branca et al ${ }^{107}$ fabricated iron oxide NPs functionalized with the LHRH targeting ligand, which could then be visualized in vivo by high-resolution (HR) $3 \mathrm{He}$ MRI. When tested in vivo in mice injected with breast MDA-MB-231 adenocarcinoma or prostate cancer PC3 cells, this system could efficiently detect pulmonary micrometastases with molecular specificity 24-48 h after injection.

Jiang et al ${ }^{108}$ designed an RGD-conjugated 3-(aminopropyl)trimethoxysilane-modified USPION and used a $3.0 \mathrm{~T}$ clinical MR scanner to non-invasively characterize the $\alpha_{v} \beta_{3}$ expression pattern in animals with lung cancer. The two tumor models used, A549 and 3LL lung cancer tumors, were shown to have different $\alpha_{\mathrm{v}} \beta_{3}$ expression patterns in MR images. For example in A549 tumors, $\alpha_{\mathrm{v}} \beta_{3}$ expression was shown throughout the tumor, as evidenced by a homogenous decrease of signal intensity. Histological examinations of A549 tumor also revealed the expression of $\alpha_{v} \beta_{3}$ in both the tumor vessel and tumor cells. By contrast, in the $3 \mathrm{LL}$ tumor, the signal reduction was not homogenous throughout the tumor, and the majority of signal was distributed in the tumor periphery and some central regions. In these tumors, SP staining demonstrated that $\alpha_{v} \beta_{3}$ is expressed in the neovasculature. In a similar approach, Zhang et al ${ }^{109}$ fabricated a highly MR-sensitive magnetite nanocluster (MNC) probe by clustering SPIONs (each $6.5 \mathrm{~nm}$ in size) with 
(Mal)mPEG-PLA copolymer and functionalizing the resulting MNC with $\mathrm{c}(\mathrm{RGDyC})$ peptide (hydrodynamic size of $85 \mathrm{~nm}$ ). The engineered clusters were found to be highly sensitive $\left(r^{2}=\right.$ $464.94 \mathrm{~s}^{-1} \mathrm{mM}^{-1}$ ) and specific for $\alpha_{\mathrm{v}} \beta_{3}$-positive non-small cell lung carcinoma cell lines A549 and H1299. Further studies in athymic BALB/c nude mice implanted with H1299 cells $\left(4 \times 10^{6}\right)$ under the left limb, demonstrated that both nascent $\left(35 \pm 6.6 \mathrm{~mm}^{3}\right)$ and large tumors $\left(256 \pm 22.3 \mathrm{~mm}^{3}\right)$ can be targeted by engineered clusters and imaged by MRI. Moreover, the MR signal decrease was highly correlated with microvessel density and $\alpha_{\mathrm{v}} \beta_{3}$ expression levels at different growth stages, as assessed by immunohistochemical studies.

\section{Colorectal cancer}

In the United States, colorectal cancer is the third most prevalent type of cancer, accounting for $8 \%$ in men and $9 \%$ in women of all cancer-related deaths in $2015 .{ }^{1}$ The development of colorectal cancers is very slow and takes several years in most cases. This process usually starts with the formation of a non-cancerous benign polyp on the inner lining of the colon or rectum. There are two main types of polyps, hyperplastic and adenomatous, with the latter potentially resulting in cancer. Early detection is necessary to identify polyps either before they become cancerous or at the early stages, when they respond better to therapy. ${ }^{110}$ Furthermore, since survival is significantly better in non-metastatic colorectal cancer (90\% 5-year survival), early detection is of paramount importance.

Toma et al $^{111}$ have conjugated Ferumoxide SPIONs with $\mathrm{mAb}$ A7, which specifically interacts with the glycoprotein on the surface of WiDr human colonic carcinoma cell line. ${ }^{112}$ The binding activity of the conjugates was almost equal to the free mAbs. The authors tested the accumulation of this NP system in nude mice with xenograft tumors. The mAb A7-conjugated SPIONs accumulated in tumors to a greater extent than control SPIONs conjugated with non-specific IgGs. The SPIONs were cleared from plasma in a linear rate. In T2-weighted MR images of xenografts, signal intensity decreased at the tumor margin upon injection of A7-conjugated SPIONs.

In certain cases, dual mode contrast agents can be employed for coupling two imaging modalities for detection of cancer. $\mathrm{He}$ et $\mathrm{al}^{113}$ used bifunctional PEG-NHS ester disulfide (NHS-PEG-S-S-PEG-NHS) linkers for conjugating $\mathrm{Fe}_{2} \mathrm{O}_{3} @ \mathrm{Au}$ core@shell NPs with three different lectins - concanavalin A (ConA), Ricinus communis agglutinin I (RCA), WGA - for dual T2-weighted MRI and CT imaging. The Fabricated system was stable in different solutions (water, PBS and culture media) and exerted no toxic effects against the cells. After $2 \mathrm{~h}$ of incubation with SW620 cells, RCA- and WGA-bound NPs displayed strong binding affinities, exceeding those of Con A-conjugated NPs. This result confirmed that SW620 cells express high levels of glycoconjugates containing Gal and $N$-acetyl- $\beta$-D-glucosaminoside glycosyl complexes, as shown previously. ${ }^{114}$ Not only did the NPs demonstrate the capability of dual-mode MR and CT phantom imaging in vitro in SW620 cells, but they also successfully detected subcutaneous colorectal tumors (SW620) in BALB/C nude mice, a tumor model which is extensively used for mimicking the intraperitoneal metastasis of colorectal cancers.
The multifunctionality of NPs allows for simultaneous therapy and imaging. In one such study, gold-iron oxide hybrid NPs were functionalized with A33scFv. ${ }^{115}$ This antibody fragment is known to target the A33 antigen, which is expressed by the majority of primary and metastatic colorectal tumors. ${ }^{116}$ The A33scFv-targeted NPs were selectively internalized by colorectal SW1222 xenograft tumors over non-A33-expressing cells. Subsequently, these tumors underwent laser-assisted photothermal treatment $\left(808 \mathrm{~nm}, 5 \mathrm{~W}^{-1} \mathrm{~cm}^{-2}\right)$ which adversely affected more than $65 \%$ of A33-expressing SW1222 cells by damaging the ECM and cytoplasmic acidophilia. Moreover, the hybrid NPs could be selectively used to visualize subcutaneous A33-expressing xenograft tumors by MRI.

In another study incorporating a dual drug delivery/MR imaging probe, Zou et al $^{117}$ conjugated PEG-coated SPIONs (10 nm core) with HuCC $49 \Delta \mathrm{C}_{\mathrm{H}} 2$ antibody against TAG-72 antigen and 5-FAM fluorophore (final size of $44 \mathrm{~nm}$ ) for simultaneous MR and fluorescence imaging as well as $\mathrm{pH}$-dependent intracellular (lysosomal) drug release. In addition, the anticancer agents DOX, azido-DOX, MI-219, and 17-DMAG were encapsulated within these NPs. Conjugation with the antibody enhanced targeting to LS174T cells, as shown by fluorescent imaging and confirmed by MR imaging and Prussian blue staining. The targeted NPs had a T2 value of $55.5 \pm 2.6 \mathrm{~ms}$ in LS174T cells compared to $117.3 \pm 1.8 \mathrm{~ms}$ for non-targeted NPs. Among the tested encapsulated drugs, all except azido-DOX showed $\mathrm{pH}$-dependent release. The HuCC $49 \Delta \mathrm{C}_{\mathrm{H}}$ 2-targeted SPIONs had a higher efficacy in delivery of DOX to LS174T cells than non-specific IgG-targeted SPIONs (IC50 of $1.44 \mu \mathrm{M}$ vs. $0.44 \mu \mathrm{M}$ ).

\section{Cervical cancer}

Squamous cell carcinoma and adenocarcinoma are the two most common types of cervical cancer. The majority of cervical cancers are believed to arise from chronic human papillomavirus (HPV) infections. In most cases, cervical cancer can be treated if detected at early stages. Both cell penetrating peptides and small ligands have been used for targeting of cervical cancer.

$\gamma$-Amino-proline-derived membrane translocating peptides are relatively well-internalized into HeLa and COS- 1 cells, ${ }^{118}$ are less toxic than $\mathrm{TAT}^{118}$ and more stable against protease degradation owing to the $\gamma$-peptide skeleton. In a study by Cavalli et al, ${ }^{119}$ SPIONs were conjugated with $\gamma$-amino-prolinederived cell-penetrating peptide, which drastically increased the internalization of targeted SPIONs into HeLa cells compared to the analogous TAT-SPION conjugate. The internalization of NPs was also confirmed by confocal microscopy. This high cellular uptake decreased the T2 of labeled cells relative to control cells. Since the peptide was also labeled with 5(6)-carboxyfluorescein (CF) dye, these NPs combine fluorescent and magnetic properties in one construct and can be used for multimodal imaging using both MRI and confocal laser microscopy.

Sun and coworkers ${ }^{84}$ have developed PEG-coated SPIONs targeted with folate for detection of HeLa cells overexpressing FR. After $4 \mathrm{~h}$ incubation with HeLa cells, targeted NPs were internalized 12-fold more than PEG- or dextran-coated NPs. The preferential targeting of FR was shown in MR phantom imaging 
by a significant contrast enhancement in HeLa cells and not in non-FR expressing osteosarcoma MG-63 cells. Furthermore, the specificity of NPs to the FR was also compared in competitive binding studies in the presence of free folic acid. The interchain hydrogen bonding within PEG chains enhances the stability of the coating.

In addition to its use in breast cancer (discussed earlier), MTX $^{85}$ has been used as a small-molecule targeting ligand for MRI detection of HeLa cervical cancer cells. Another targeting ligand used for detection of cervical cancer is hyaluronic acid, which recognizes the $\mathrm{CD} 44$ receptor overexpressed on the surface of cancer cells. ${ }^{120} \mathrm{Li}$ et a ${ }^{121}$ modified hydrothermally synthesized polyethylenimine (PEI)-stabilized $\mathrm{Fe}_{3} \mathrm{O}_{4} \mathrm{NPs}$ with FITC and hyaluronic acid (15-16 nm). The targeted NPs were successfully taken up by CD44-overexpressing HeLa cells and could also be used in vivo for detection of tumors in a HeLa tumor-bearing mouse model.

SPIONs are sometimes coupled with other delivery agents to increase the efficacy of drug delivery and imaging by combining the advantages of both modalities. Liao et al ${ }^{122}$ employed polymeric amphiphilic octadecyl-quaternized lysine-modified chitosan (OQLCS) liposomes (PLs) conjugated with folate to coat SPIONs (8-10 nm core). HeLa cells overexpressing FR were shown to effectively internalize the folate-PLs-coated SPIONs. The intracellular iron density was higher in cells incubated with folate-PLs-coated SPIONs than those incubated with folate-free PLs-coated SPIONs. Overall, the folate-PLscoated SPIONs also produced better T2-weighted MR images than folate-free PLs-coated SPIONs.

\section{Ovarian cancer}

Ovarian cancer has received relatively less attention compared to other types of cancer. One of the main markers for ovarian cancer is the ovarian cancer-specific transmembrane glycoprotein antigen, MUC1, which can be targeted by C595 antibody. ${ }^{123}$ Since the $\mathrm{C} 595 \mathrm{mAb}$ has anticancer activity, it can have dual functions in both targeting and treating tumors. SPIONs were thus conjugated with C595 mAb to produce contrast agents for detection of ovarian cancer. ${ }^{124}$ The nanoprobe reduced the signal intensity in MUC1-expressing OVCAR3 cells by $95 \%$, compared with their non-targeted counterparts. Moreover, the affinity of NPs for OVCAR3 cells was 5 times higher than that of non-targeted NPs. C595-conjugated SPIONs accumulated twice as much in the tumor site than in other organs. While the reduction in signal intensity was $56 \%$ for C595-conjugated SPIONs, it was only $10 \%$ for non-targeted SPIONs. Moreover, the NPs exerted selective toxicity against ovarian cancer in vivo. The main disadvantage of these NPs was the dramatic uptake by RES $24 \mathrm{~h}$ after injection; however, in special cases, this feature can also be suitable for detection of liver and spleen tumors.

Affibodies are antibody-mimetic molecules that can bind to a large number of target proteins at the same time. In a study by Gao et $\mathrm{al}^{125}$ Her2 affibodies were used to label maleimidefunctionalized NIR quantum dots (a core of $5 \mathrm{~nm}$ with an emission wavelength of $\sim 800 \mathrm{~nm}$ ) or iron oxide NPs (core of $15 \mathrm{~nm}$ ). Her2 is overexpressed in a number of cancers such as breast, ovarian, lung, and gastric cancers. These conjugates were then shown to be highly specific for Her2-expressing ovarian SKOV3 cells and tumors. While the targeted quantum dots could be used for fluorescent imaging of SKOV3 tumors in vivo and ex vivo $4 \mathrm{~h}$ post-injection, treatment of cells in vitro with affibody-labeled NPs produced significant cellular MRI contrast compared to the identical cells treated with untargeted PEGcoated iron oxide NPs. However, only a slight decrease in MRI signal was observed upon MR imaging of SKOV3 tumor in vivo, which was attributed to the low tumor uptake of affibody-labeled iron oxide NPs or the low sensitivity of MRI.

Other potentially interesting molecular targets that can be explored for imaging and early detection of ovarian cancer include CD276, ${ }^{126}$ CD24, HE4, CD9, GA733-2 as well as cytokeratins 7, 8, 18 , and $19 .{ }^{127}$

\section{Other studies}

Suwa et al $^{128}$ coated SPIONs $(13.2 \pm 1.9 \mathrm{~nm})$ with mAbs directed against EGFR. EGFR is often over-expressed in esophageal squamous cell carcinoma. The conjugated mAbs retained their bioactivity after binding to the NP surface. The contrast enhancement in T2 images was shown in the EGFR-expressing cell line TE8 compared to the non-EGFR-expressing cell line H69. The subsequent in vivo studies in athymic rats bearing TE8 or H69 tumors also confirmed the EGFR-specific MRI contrast specificity. The magnetic NPs were found to localize to the lysosomes upon internalization, as shown by electron microscopy studies.

Carbohydrates can also be used for detection of lectin receptors present on the surface of cancer cells. In one study, ${ }^{129}$ the authors used carbohydrates to synthesize glyco-NPs for detection of cancer cells. Furthermore, they intended to quantitatively assess the carbohydrate binding signature of cancer cells. The authors used an array of magnetic NPs, each functionalized with a specific sugar (Figure 10), and showed that the NP array can differentiate malignant from normal cells, closely related isogenic tumor cells, and cells with different metastatic potential based on the unique lectin profile of the different cells (Figure 11). Detection was based on the different MRI signatures of the glycoconjugates.

This study also has implications in characterizing the "glycocode" of different cancer cells, contributing to the overall understanding of carbohydrates in cancer. Biological information is encoded and stored within the varied structures of cell-surface carbohydrates, which are involved in cell differentiation, migration to specific tissues, cell adhesion, microbial pathogenesis, and recognition by the immune system. ${ }^{130}$ In other words, the glycocode can be the key to devising new diagnostic and therapeutic modalities.

In an effort to detect leukemia cells in blood samples, Herr et al ${ }^{131}$ developed a system composed of two NPs for collection and subsequent sensing of these cells. For this purpose, SPIONs were conjugated with an aptamer selected against CCRF-CEM acute leukemia cells. Aptamer-modified magnetic NPs were used for extraction of target cells, while fluorescent NPs conjugated with the same aptamer were used for sensitive detection of cells, such that even single-aptamer binding events could be sensed. 


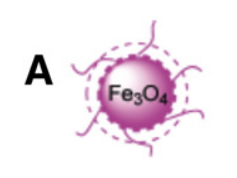

NP 1

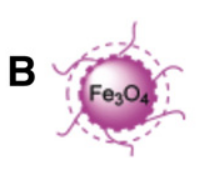

NP 1

\section{APTES \\ 1) $\left(\mathrm{C}_{2} \mathrm{H}_{5} \mathrm{O}\right)_{3} \mathrm{Si} \sim \mathrm{NH}_{2}$}
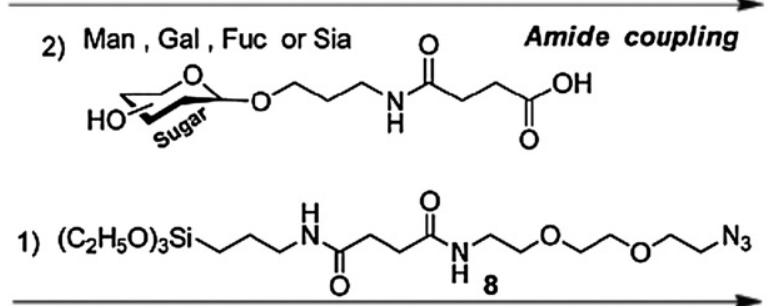

Click coupling

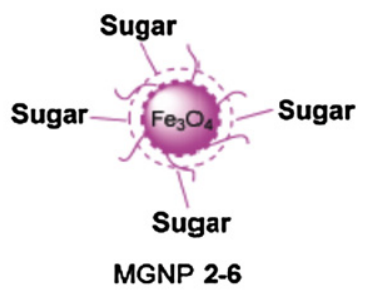

Sugar $=$

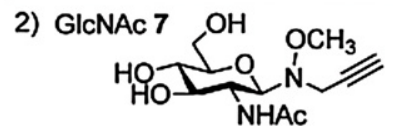

MGNP 2-6
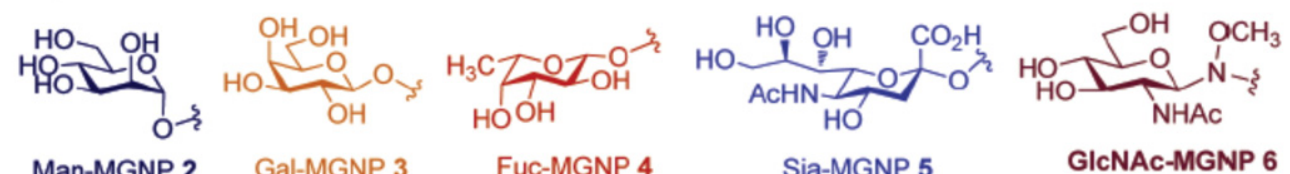

Figure 10. The synthesis of carbohydrate-functionalized magnetic NPs. Reproduced with permission. ${ }^{129}$

\section{Detection of metastases}

In any type of cancers, the minority of cancerous cells can leave the cancer site, get into the circulation (so called "circulating tumor cells") and find a new site to settle down. This phenomenon can lead to the formation of additional cancerous sites (i.e., metastasis). As the number of circulating tumor cells are rare in the first stage of metastasis, early detection and analysis of these circulating cells in clinic are of crucial importance. Recent reports demonstrated promising results in use of various types of NPs (more specifically magnetic NPs) for detection of circulating tumor cells. These reports were completely reviewed by Bhana et al. ${ }^{132}$

Due to the similarity between normal and metastatic lymph nodes, devising non-invasive methods for detection of metastatic lymph nodes is a challenging task. To design SPIONs which could be specifically internalized by immune cells, such as macrophages and dendritic cells within lymph nodes, Vu-Quang et al $^{133}$ coated SPIONs with mannan polysaccharide (hydrodynamic size of $46.2 \pm 1.9 \mathrm{~nm}$ ). Having a high D-mannose content, mannan can bind mannose receptors on activated macrophages as well as dendritic cells. Mannan-coated SPIONs were significantly internalized by freshly-isolated peritoneal macrophages, as confirmed by Prussian blue and fluorescence staining, which led to a significant improvement in contrast enhancement upon MR phantom imaging $2 \mathrm{~h}$ post-treatment. When administered to mice, these conjugates decreased the relative signal intensity between popliteal lymph nodes and muscles at $1 \mathrm{~h}$ post-injection and for up to $24 \mathrm{~h}$.

In another study, a metastatic lymph node model was created in BALB/c mice with subcutaneous injection of $10^{7}$ malignant 4T1 breast cancer cells. Mannan-coated SPIONs could differentiate normal from metastatic lymph nodes. It was hypothesized that metastatic lymph nodes do not possess antigen presenting cell-related functions and do not internalize mannan-coated SPIONs to the extent that normal lymph nodes do. In normal lymph nodes, there was a uniform reduction in signal intensity throughout the whole region; whereas in metastatic lymph nodes, the MR signal intensity was almost unchanged after administration of contrast agent. In another study, ${ }^{134}$ TCL SPIONs were employed for MRI detection of lymph node metastasis in C57BL/6 mice subcutaneously injected with B16F1 human melanoma cells. In the majority of the nodes, the enhancement pattern was consistent with the pathology. In another approach, Vu-Quang et $\mathrm{al}^{135}$ synthesized SPIONs $(15.7 \pm 6.3 \mathrm{~nm}$ core $)$ functionalized with $\beta$-glucan, which can activate macrophages by binding to $(1,3)-\beta-D-g l u c a n$ receptor or Dectin-1 receptor. These SPIONs could target macrophages surrounding the metastatic regions of the liver. Upon MR imaging in the metastatic mouse liver model (created by injection of CT-26 colorectal carcinoma cells into a hemi-spleen), the preferential uptake of targeted SPIONs within the metastases differentiated them from the tumor cells, which did not internalize these NPs.

In order to detect the metastatic cells, Peiris et al, ${ }^{136}$ designed chain shaped iron oxide NPs consisting of four iron oxide nanosphere connected to each other in aligned direction. The chain shaped iron oxide NPs were functionalized by cyclic tripeptide arginine, glycine, glycine, aspartic acid that specifically attach to $\alpha_{\mathrm{v}} \beta_{3}$ integrin. As the $\alpha_{\mathrm{v}} \beta_{3}$ integrin is required for metastatic site transition, its presence on the surface of cells can be as used as potential diagnostic sign of metastasis cells. Because of their multisite attachment on the $\alpha_{v} \beta_{3}$ integrin, the chain shaped nanospheres showed high targeting and imaging efficacy for detection of metastatic cell in animals with liver, lung and breast cancer.

The importance of developing animal models for testing targeted contrast agents

Developing in vivo models for preclinical studies prior to testing novel contrast agents and imaging platforms on humans is essential, especially in cancers for which a suitable animal model 
A) $10^{\wedge} 5$ cells $/ \mathrm{mL}$

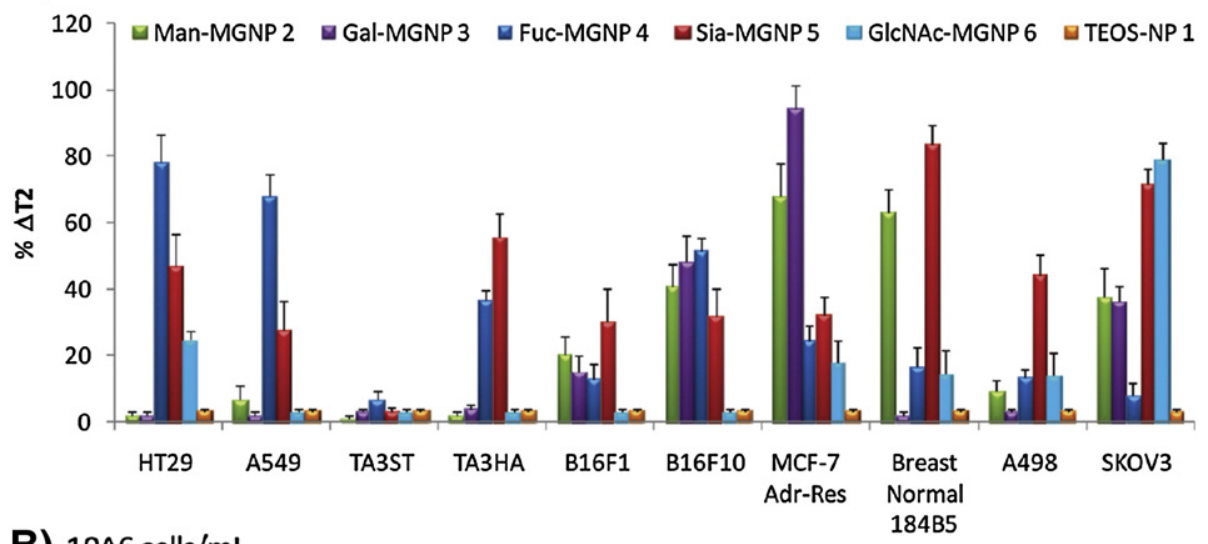

B) $10^{\wedge} 6$ cells $/ \mathrm{mL}$

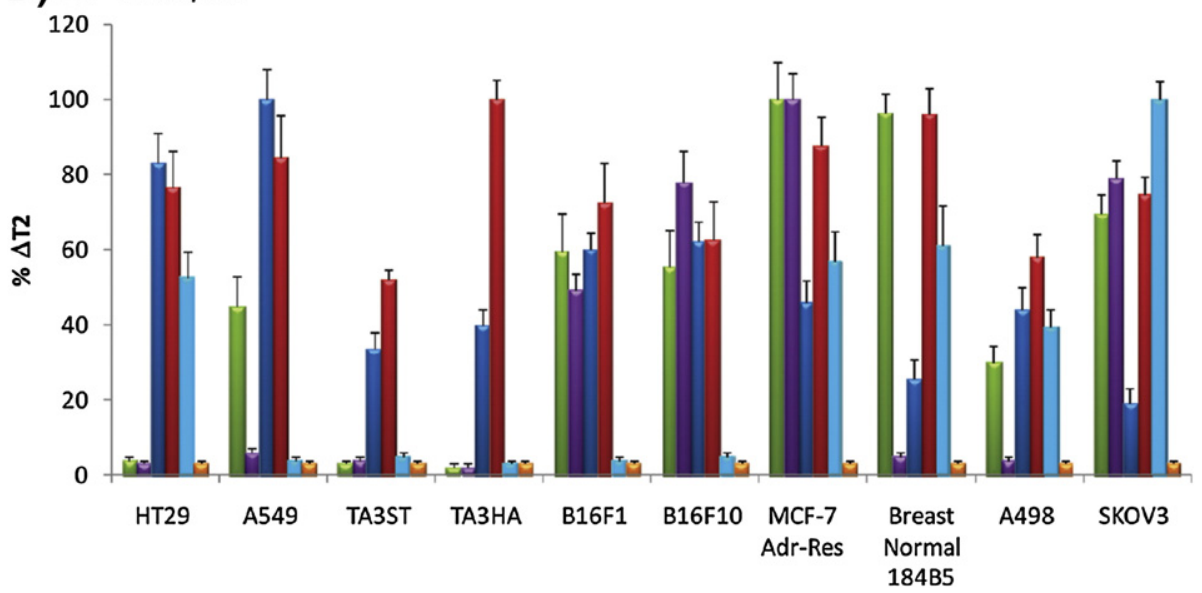

Figure 11. (A) Percentage change in T2 relaxation time after incubation of different magnetic $(\mathrm{n}=6) \mathrm{NPs}(20 \mu \mathrm{g} / \mathrm{mL})$ with the 10 cell lines in the study $\left(10^{5}\right.$ cells $/ \mathrm{mL})$. (B) Percentage change in T2 relaxation time after incubation of magnetic NPs $(20 \mu \mathrm{g} / \mathrm{mL})$ with 10 cell lines at the density of $10^{6}$ cells $/ \mathrm{mL}$. The significant difference in binding to different magnetic NPs led to the differentiation of these cell lines from each other. Reproduced with permission. ${ }^{129}$

is not readily available. As such, different groups have tried to establish animal models for certain tumor types or their metastases. For example, Ocak et a ${ }^{137}$ developed an FR-positive murine ovarian cancer cell line, which could induce widespread intraperitoneal tumors in immunocompetent mice within 4-8 weeks after implantation. The authors monitored the process of tumor development using various imaging modalities, including PET/CT in combination with a PET FR-targeting reporter and fluorescence molecular tomography (FMT) in combination with a commercial FR-targeting reporter.

Selective and specific detection of early prostate cancer metastases to bone is a challenging task for current clinical imaging techniques. In a similar effort as above, Sroka et al ${ }^{138}$ developed and established a reproducible bone metastasis xenograft model, and also found cell-surface receptors that could function as molecular imaging candidates for metastatic prostate cancer. For this purpose, bone-trophic human prostate cells (PC-3B1) were injected intracardially into SCID mice. Potential skeletal metastases were investigated. The cells were found to maintain their bone-homing phenotype for a relatively long time in culture and in vivo 3 weeks after intra-cardiac injection. PC-3 and PC-3B1 cells were subjected to transcriptomic profiling using a cancer-specific microarray. Among the 55 upregulated genes ( $\geq 1.5$ fold upregulation) in
PC-3B1 cells, only AXL, CDH11, CD34, and MET were membrane receptors expressed in human tumors. These proteins can be potentially used for targeting of bone metastasis associated with prostate cancer.

\section{Protein corona}

The "protein corona" is the layer (or composed of several layers) of proteins and other types of biomolecules that immediately covers the surface of NPs upon entry into a biological fluid, such as plasma. ${ }^{139-141}$ This is due to their high surface to volume ratio which confers a very active surface chemistry to the particles. It has been shown in several studies that the process of corona formation is very dynamic and competitive. The evolution of protein corona starts with rapid adsorption of low-affinity high-abundance proteins, some of which are later exchanged with proteins of higher affinity and lower abundance. ${ }^{142}$

The composition of proteins in the corona is largely dictated by NP size and surface chemistry, ${ }^{143}$ crystallinity ${ }^{144}$ and hydrophobicity ${ }^{145}$ as well as other parameters such as plasma concentration $^{146}$ and temperature. ${ }^{147,148}$ It is now being 
increasingly accepted that the type of disease in human patients can also change the plasma composition which mirror significant variation in structures of protein corona (i.e., amount, type, and conformation of the associated proteins in the corona composition). ${ }^{149-152}$ For example, it was demonstrated that the cellular response (e.g., cellular toxicity, apoptosis, uptake, reactive oxygen species production, lipid peroxidation and nitrogen oxide levels) to the exact type of graphene oxide nanosheets, but with coronas from different patients (including hypofibrinogenemia, blood cancer, thalassemia major, thalassemia minor, rheumatism, fauvism, hypercholesterolemia, diabetes), was considerably different. ${ }^{149}$ The key message of this patient specific protein corona is that specific design should be considered for NPs, which are intended to be used in patients with different diseases, to get safe and efficient targeting efficacy.

\section{Complications of protein corona for delivery systems and MRI contrast agents}

As protein corona is the first contact point of an NP with the cells and other biological species, the decoration of corona can have a significant impact on the interactions of NPs with biological systems. The proteins with highest affinity or the so called hard corona mainly mediate the interaction of a given NP with biological components. ${ }^{153}$

As such, the corona decoration can interfere with the engineered functions of NPs. The protein corona has been shown to offset the targeting capability of functional ligands conjugated to NPs by up to $96 \%{ }^{154,155}$ This shows that majority of successful in vitro nanoparticulate systems may have very low in vivo efficacies. In addition, these NPs which are mainly used for target-specific applications could be internalized by other sites (as results of mis-targeting), such as brain tissue, and cause severe unwanted cytotoxicity. ${ }^{156}$ In order to overcome this issue, one should be able to control the decoration of protein corona (mainly by adjusting physicochemical properties of NPs) or use proper coatings that inhibit protein absorption to the surface of NPs (e.g., zwitterionic coatings). ${ }^{157}$

Besides mis-targeting, adsorption of proteins has the capability to largely change the relaxivity of contrast agents in MR imaging. Amiri et al ${ }^{158}$ evaluated the effects of protein adsorption from $10 \% \mathrm{v} / \mathrm{v}$ FBS on the magnetic properties of NPs and their MRI contrast efficiency. For this purpose, two series of SPIONs were synthesized, which differed in thickness and functional groups (i.e., surface charges) of the dextran surface coating. These different physiochemical characteristics resulted in the generation of dissimilar protein coronas. Only plain NPs retained their original relaxivity in the presence of protein corona. The relaxivity of negatively charged SPIONs mildly increased upon introduction into biological fluid and formation of protein corona, while that of positively charged SPIONs considerably reduced in the same conditions. In vitro MRI experiments also confirmed the relaxometry findings. Therefore, the protein corona is an additional criterion that must be taken into account in the NP engineering process for in vivo imaging and delivery applications.

Apart from influencing the drug delivery and MRI efficiency, there are a couple of other concerns that are important considerations for NP engineering, though they might not be of immediate relevance to targeting. NPs can induce conformational changes in the proteins they come in contact with. For example, gold NPs have been shown to induce reversible conformational changes in both secondary and tertiary structures of BSA. ${ }^{159}$ Furthermore, Mahmoudi et al ${ }^{160}$ have reported that the interaction of SPIONs with iron-saturated human transferrin can irreversibly open its compact conformation. NP-induced protein denaturation and unfolding can lead to protein aggregation and/or presentation of new epitopes, which can hypothetically cause conditions such as amyloidosis ${ }^{161}$ or immunological reactions ${ }^{162}$ and inflammation. ${ }^{161}$

Rational engineering of NP surface can help reduce the denaturation of interacting proteins. For example, while anionic NPs inhibit the enzymatic activity of $\alpha$-chymotrypsin by denaturing its secondary structure, ${ }^{163}$ functionalizing NPs with oligo(ethylene glycol) (OEG) significantly reduces $\alpha$-chymotrypsin denaturation. ${ }^{164}$ Indeed, the activity of enzyme can be specifically modulated by NPs, since chymotrypsin interaction with the anionic NPs increased the catalytic activity of the enzyme for cationic substrates by 3 fold, while the activity was reduced for neutral and anionic substrates by 50 and $95 \%$, respectively. ${ }^{163}$ Interestingly, 2-(10-mercaptodecyl)malonic acid-functionalized gold NPs have been shown to facilitate the refolding of thermally denatured cationic proteins such as chymotrypsin, papain and lysozyme. ${ }^{165}$

Therefore, in the future challenge called "safety by design", the ultimate purpose will be to tailor the various physiochemical properties of a given NP such that the protein NP interactions can be controlled and/or at least predicted to some extent.

\section{Potential advantages of protein corona}

On the other hand, predictable enrichment of specific proteins can come in handy in targeting of cells and tissues in delivery and imaging applications. For example, albumin and apolipoprotein adsorption leads to higher NP circulation times in plasma, ${ }^{166}$ while opsonins such as fibrinogen, immunoglobulins and complement proteins promote NP phagocytosis by cells of the RES. ${ }^{167}$ Fibrinogen can promote the recognition of foreign entities by monocytes, macrophages, and neutrophils, for example through binding to CD11/CD18 on phagocytes. ${ }^{168,169}$ As another example, the cellular uptake of magnetite NPs by macrophages was higher when lung surfactant protein A adsorbed on their surface in comparison with NPs precoated with BSA. ${ }^{170}$ The NPs harboring opsonin proteins in their corona can potentially be promising candidates for delivery and imaging in the RES. For a number of similar examples on the effect of adsorbed proteins on targeting and cellular uptake, please refer to this book chapter. ${ }^{171}$

Furthermore, the formation of protein corona is an inherent physiological strategy to deal with the toxicity of foreign substances. There are an increasing number of papers showing that the protein corona reduces the toxicity of NPs in biological systems. For instance, such mitigation of cytotoxicity has been noted with silica and polystyrene $\mathrm{NPs}^{172}$ as well as graphene oxide. $^{173}$ 


\section{Complying with protein corona in drug delivery}

Provided the fact that protein corona formation is an inevitable phenomenon in vivo, three paradigms have been proposed in the delivery context. ${ }^{171}$ In the first modality, NPs can be rendered non-fouling to circumvent the formation of protein corona. ${ }^{157}$ In this approach, NPs are usually coated with hydrophilic polymers, such as $\mathrm{PEG}^{174}$ or are functionalized with zwitterionic groups such as amino acids and polybetaines. In one such study, a number of zwitterionic gold NPs were produced which could resist the adsorption of serum proteins in moderate concentrations, and did not develop a hard corona at physiological serum levels. ${ }^{157}$

In the second approach, some groups have actually started to exploit the protein corona for drug loading and release. For example in one study, ${ }^{175}$ both negatively charged oligonucleotides and positively charged doxorubicin were loaded in the protein corona of cetyltrimethylammonium bromide (CTAB)coated gold nanorods. The holding capacity of protein corona on the nanorods was significantly higher than the covalent conjugation of therapeutics on NP surfaces. As the temperature has crucial effect on the protein corona composition, ${ }^{147,176}$ the release of cargo could be changed by photo-induced plasmonic heating of gold nanorods.

In the third approach, the formed protein corona itself can be actively employed for lending targeting capabilities to the delivery system. For materialization of such a strategy, the composition of protein corona on the surface of specific NPs with fully characterized surface chemistry must be thoroughly determined, differentiating the proteins with highest abundance or highest affinity on NP surface. ${ }^{171}$ In this regard, for example, NPs with a corona enriched in transferrin, can be used for targeting of transferrin over-expressing cells such as BT20, MCF7, BT549, and HBL100. ${ }^{177}$ Similarly, most hydrophobic NPs have been shown to enrich apolipoprotein A-I in their corona, and they can be potentially used for drug delivery to neurons, as apolipoprotein A-I interacts with low density lipoprotein (LDL) and scavenger receptors in the brain capillary endothelial cells. ${ }^{178}$ It is also noteworthy that scavenger receptors are overexpressed in PC3 prostate carcinoma cell line as well as in the liver and steroidogenic glands, intestine, and placenta, and in cells such as macrophages and endothelial cells. ${ }^{179}$ Progressive increase in our understanding of NP-protein interactions will eventually help us overcome the challenges and design futuristic highly specific and characterized NP systems for delivery and imaging applications.

\section{Conclusions and outlook}

Despite the fact that targeted SPIONs have great potential for theranostics of cancer and possess an acceptable safety margin for clinical applications, except for most attractive cancer types, only a few SPION based platforms have been fabricated. Furthermore, possibly the whole field of targeted imaging and detection suffers from the lack of very specific and versatile tumor markers and also targeting moieties that can recognize such targets. Most tumor markers currently such as FR and integrins are unspecifically overexpressed by a diverse panel of tumors. Small antibody fragments such as ScFvs can be valuable functional targeting ligands for fabrication of NP-based imaging systems with regard to highest specificity and relatively small size, however, the complexity of fabrication and potentially the difficulty in obtaining approval from regulatory agencies can hinder their clinical applications. Researchers should also make comprehensive progress toward removing adverse effects of protein corona in cancer targeting. More specifically, the personalized protein corona should be considered in fabrication of targeted NPs. Since early diagnosis and detection of specific cancer cells in vivo have great implications in patient treatment and prognosis, more studies need to be focused on fabrication of NP-based systems for cancer imaging, while in parallel, cancer genomic and proteomic screenings can be used for identification and characterization of specific tumor markers for explicit differentiation of specific tumor types.

\section{References}

1. Siegel RL, Miller KD, Jemal A. Cancer statistics, 2015. CA Cancer J Clin 2015;65:5-29.

2. Frush DP, Applegate K. Computed tomography and radiation: understanding the issues. J Am Coll Radiol 2004;1:113-9.

3. Deroose CM, De A, Loening AM, Chow PL, Ray P, Chatziioannou AF, et al. Multimodality imaging of tumor xenografts and metastases in mice with combined small-animal PET, small-animal CT, and bioluminescence imaging. J Nucl Med 2007;48:295-303.

4. Laurent S, Saei AA, Behzadi S, Panahifar A, Mahmoudi M. Superparamagnetic iron oxide nanoparticles for delivery of therapeutic agents: opportunities and challenges. Exp Opin Drug Deliv 2014;1-22.

5. Berensmeier S. Magnetic particles for the separation and purification of nucleic acids. Appl Microbiol Biotechnol 2006;73:495-504.

6. Laurent S, Dutz S, Häfeli UO, Mahmoudi M. Magnetic fluid hyperthermia: focus on superparamagnetic iron oxide nanoparticles. Adv Colloid Interf Sci 2011;166:8-23.

7. Saei AA, Barzegari A, Heydari Majd M, Asgari D, Omidi Y. Fe3O4 nanoparticles engineered for plasmid DNA delivery to Escherichia coli. J Nanoparticle Res 2014;16.

8. Rahman M, Saei AA, Amiri H, Mahmoudi M. Biomedical applications of superparamagnetic nanoparticles in molecular scale. Curr Org Chem 2015;19:982-90.

9. Mahmoudi M, Sant S, Wang B, Laurent S, Sen T. Superparamagnetic iron oxide nanoparticles (SPIONs): development, surface modification and applications in chemotherapy. Adv Drug Deliv Rev 2011;63:24-46.

10. Mahmoudi M, Hosseinkhani H, Hosseinkhani M, Boutry S, Simchi A, Journeay WS, et al. Magnetic resonance imaging tracking of stem cells in vivo using iron oxide nanoparticles as a tool for the advancement of clinical regenerative medicine. Chem Rev 2010;111:253-80.

11. Wang Y-XJ. Superparamagnetic iron oxide based MRI contrast agents: current status of clinical application. Quant Imaging Med Surg 2011;1:35.

12. Ahmad T, Bae H, Rhee I, Chang Y, Lee J, Hong S. Particle size dependence of relaxivity for silica-coated iron oxide nanoparticles. Curr Appl Phys 2012;12:969-74.

13. Huang J, Zhong X, Wang L, Yang L, Mao H. Improving the magnetic resonance imaging contrast and detection methods with engineered magnetic nanoparticles. Theranostics 2012;2:86.

14. Y-w Jun, Huh Y-M, J-s Choi, Lee J-H, Song H-T, Kim S, et al. Nanoscale size effect of magnetic nanocrystals and their utilization for cancer diagnosis via magnetic resonance imaging. J Am Chem Soc 2005;127:5732-3.

15. Xie J, Chen K, Huang J, Lee S, Wang J, Gao J, et al. PET/NIRF/MRI triple functional iron oxide nanoparticles. Biomaterials 2010;31:3016-22.

16. Petros RA, DeSimone JM. Strategies in the design of nanoparticles for therapeutic applications. Nat Rev Drug Discov 2010;9:615-27. 
17. Fang J, Nakamura H, Maeda H. The EPR effect: unique features of tumor blood vessels for drug delivery, factors involved, and limitations and augmentation of the effect. Adv Drug Deliv Rev 2011;63:136-51.

18. Moore A, Marecos E, Bogdanov Jr A, Weissleder R. Tumoral distribution of long-circulating dextran-coated iron oxide nanoparticles in a rodent model 1. Radiology 2000;214:568-74.

19. Byrne JD, Betancourt T, Brannon-Peppas L. Active targeting schemes for nanoparticle systems in cancer therapeutics. Adv Drug Deliv Rev 2008;60:1615-26.

20. Iagaru A, Chen X, Gambhir SS. Molecular imaging can accelerate antiangiogenic drug development and testing. Nat Clin Pract Oncol 2007;4:556-7.

21. Alexiou C, Arnold W, Klein RJ, Parak FG, Hulin P, Bergemann C, et al. Locoregional cancer treatment with magnetic drug targeting. Cancer Res 2000;60:6641-8.

22. Rosen JE, Chan L, Shieh D-B, Gu FX. Iron oxide nanoparticles for targeted cancer imaging and diagnostics. Nanomedicine 2012;8:275-90.

23. Allen TM. Ligand-targeted therapeutics in anticancer therapy. Nat Rev Cancer 2002;2:750-63.

24. Gu FX, Karnik R, Wang AZ, Alexis F, Levy-Nissenbaum E, Hong S, et al. Targeted nanoparticles for cancer therapy. Nano Today 2007;2:14-21.

25. Chester KA, Hawkins RE. Clinical issues in antibody design. Trends Biotechnol 1995;13:294-300.

26. Tiefenauer LX, Kuehne G, Andres RY. Antibody-magnetite nanoparticles: in vitro characterization of a potential tumor-specific contrast agent for magnetic resonance imaging. Bioconjug Chem 1993;4:347-52.

27. Brannon-Peppas L, Blanchette JO. Nanoparticle and targeted systems for cancer therapy. Adv Drug Deliv Rev 2012;64:206-12.

28. Kuus-Reichel K, Grauer L, Karavodin L, Knott C, Krusemeier M, Kay $\mathrm{N}$. Will immunogenicity limit the use, efficacy, and future development of therapeutic monoclonal antibodies? Clin Diagn Lab Immunol 1994;1:365-72.

29. Vigor KL, Kyrtatos PG, Minogue S, Al-Jamal KT, Kogelberg H, Tolner B, et al. Nanoparticles functionalised with recombinant single chain $\mathrm{Fv}$ antibody fragments (scFv) for the magnetic resonance imaging of cancer cells. Biomaterials 2010;31:1307-15.

30. Tuerk C, Gold L. Systematic evolution of ligands by exponential enrichment: RNA ligands to bacteriophage T4 DNA polymerase. Science 1990;249:505-10.

31. Sefah K, Shangguan D, Xiong X, O’Donoghue MB, Tan W. Development of DNA aptamers using Cell-SELEX. Nat Protoc 2010;5:1169-85.

32. Jayasena SD. Aptamers: an emerging class of molecules that rival antibodies in diagnostics. Clin Chem 1999;45:1628-50.

33. Huang Y-F, Chang H-T, Tan W. Cancer cell targeting using multiple aptamers conjugated on nanorods. Anal Chem 2008;80:567-72.

34. Zhao Z, Xu L, Shi X, Tan W, Fang X, Shangguan D. Recognition of subtype non-small cell lung cancer by DNA aptamers selected from living cells. Analyst 2009;134:1808-14.

35. Li X, Zhang W, Liu L, Zhu Z, Ouyang G, An Y, et al. In vitro selection of DNA aptamers for metastatic breast cancer cell recognition and tissue imaging. Anal Chem 2014;86:6596-603.

36. Kim MY, Jeong S. In vitro selection of RNA aptamer and specific targeting of ErbB2 in breast cancer cells. Nucleic Acids Ther 2011;21:173-8.

37. Li S, Xu H, Ding H, Huang Y, Cao X, Yang G, et al. Identification of an aptamer targeting hnRNP A1 by tissue slide-based SELEX. J Pathol 2009;218:327-36.

38. Song Y, Zhu Z, An Y, Zhang W, Zhang H, Liu D, et al. Selection of DNA aptamers against epithelial cell adhesion molecule for cancer cell imaging and circulating tumor cell capture. Anal Chem 2013;85:4141-9.

39. Kimura RH, Cheng Z, Gambhir SS, Cochran JR. Engineered knottin peptides: a new class of agents for imaging integrin expression in living subjects. Cancer Res 2009;69:2435-42.
40. Li M, Song L, Qin X. Glycan changes: cancer metastasis and anticancer vaccines. $J$ Biosci 2010;35:665-73.

41. Singh A, Dilnawaz F, Sahoo SK. Long circulating lectin conjugated paclitaxel loaded magnetic nanoparticles: a new theranostic avenue for leukemia therapy. PLoS One 2011;6e26803.

42. Wagstaff KM, Jans DA. Protein transduction: cell penetrating peptides and their therapeutic applications. Curr Med Chem 2006;13:1371-87.

43. Sudimack J, Lee RJ. Targeted drug delivery via the folate receptor. $A d v$ Drug Deliv Rev 2000;41:147-62.

44. Weitman SD, Lark RH, Coney LR, Fort DW, Frasca V, Zurawski VR, et al. Distribution of the folate receptor GP38 in normal and malignant cell lines and tissues. Cancer Res 1992;52:3396-401.

45. Reimer P, Tombach B. Hepatic MRI with SPIO: detection and characterization of focal liver lesions. Eur Radiol 1998;8:1198-204.

46. Sun S, Zeng H. Size-controlled synthesis of magnetite nanoparticles. $J$ Am Chem Soc 2002;124:8204-5.

47. Lee J-H, Huh Y-M, Y-w Jun, J-w Seo, J-t Jang, Song H-T, et al. Artificially engineered magnetic nanoparticles for ultra-sensitive molecular imaging. Nat Med 2007;13:95-9.

48. Zhao Z, Zhou Z, Bao J, Wang Z, Hu J, Chi X, et al. Octapod iron oxide nanoparticles as high-performance T2 contrast agents for magnetic resonance imaging. Nat Commun 2013;4.

49. Li Y-W, Chen Z-G, Zhao Z-S, Li H-L, Wang J-C, Zhang Z-M. Preparation of magnetic resonance probes using one-pot method for detection of hepatocellular carcinoma. World J Gastroenterol 2015;21:4275.

50. Maeng JH, Lee D-H, Jung KH, Bae Y-H, Park I-S, Jeong S, et al. Multifunctional doxorubicin loaded superparamagnetic iron oxide nanoparticles for chemotherapy and magnetic resonance imaging in liver cancer. Biomaterials 2010;31:4995-5006.

51. Pilapong C, Raiputta C, Chaisupa J, Sittichai S, Thongtem S, Thongtem T. Magnetic-EpCAM nanoprobe as a new platform for efficient targeting, isolating and imaging hepatocellular carcinoma. RSC $A d v$ 2015;5:30687-93.

52. Fortuin AS, Meijer H, Thompson LC, Witjes JA, Barentsz JO. Ferumoxtran-10 ultrasmall superparamagnetic iron oxide-enhanced diffusion-weighted imaging magnetic resonance imaging for detection of metastases in normal-sized lymph nodes in patients with bladder and prostate cancer: do we enter the era after extended pelvic lymph node dissection? Eur Urol 2013;64:961-3.

53. Israeli RS, Powell CT, Corr JG, Fair WR, Heston WD. Expression of the prostate-specific membrane antigen. Cancer Res 1994;54:1807-11.

54. Chang SS, Reuter VE, Heston W, Bander NH, Grauer LS, Gaudin PB. Five different anti-prostate-specific membrane antigen (PSMA) antibodies confirm PSMA expression in tumor-associated neovasculature. Cancer Res 1999;59:3192-8.

55. Smith-Jones PM, Vallabhajosula S, Navarro V, Bastidas D, Goldsmith SJ, Bander NH. Radiolabeled monoclonal antibodies specific to the extracellular domain of prostate-specific membrane antigen: preclinical studies in nude mice bearing LNCaP human prostate tumor. J Nucl Med 2003;44:610-7.

56. Bander NH, Trabulsi EJ, Kostakoglu L, Yao D, Vallabhajosula S, Smith-Jones P, et al. Targeting metastatic prostate cancer with radiolabeled monoclonal antibody J591 to the extracellular domain of prostate specific membrane antigen. J Urol 2003;170:1717-21.

57. Abdolahi M, Shahbazi-Gahrouei D, Laurent S, Sermeus C, Firozian F, Allen BJ, et al. Synthesis and in vitro evaluation of MR molecular imaging probes using J591 mAb-conjugated SPIONs for specific detection of prostate cancer. Contrast Media Mol Imaging 2013;8:175-84.

58. Holland JP, Divilov V, Bander NH, Smith-Jones PM, Larson SM, Lewis JS. 89Zr-DFO-J591 for immunoPET of prostate-specific membrane antigen expression in vivo. J Nucl Med 2010;51:1293-300.

59. Wang AZ, Bagalkot V, Vasilliou CC, Gu F, Alexis F, Zhang L, et al. Superparamagnetic iron oxide nanoparticle-aptamer bioconjugates for combined prostate cancer imaging and therapy. ChemMedChem 2008;3:1311-5. 
60. Cho H-S, Dong Z, Pauletti GM, Zhang J, Xu H, Gu H, et al. Fluorescent, superparamagnetic nanospheres for drug storage, targeting, and imaging: a multifunctional nanocarrier system for cancer diagnosis and treatment. ACS Nano 2010;4:5398-404.

61. Ghosh D, Lee Y, Thomas S, Kohli AG, Yun DS, Belcher AM, et al. M13-templated magnetic nanoparticles for targeted in vivo imaging of prostate cancer. Nat Nanotechnol 2012;7:677-82.

62. Gao X, Luo Y, Wang Y, Pang J, Liao C, Lu H, et al. Prostate stem cell antigen-targeted nanoparticles with dual functional properties: in vivo imaging and cancer chemotherapy. Int J Nanomedicine 2012;7:4037.

63. Serres S, Soto MS, Hamilton A, McAteer MA, Carbonell WS, Robson MD, et al. Molecular MRI enables early and sensitive detection of brain metastases. Proc Natl Acad Sci 2012;109:6674-9.

64. Krol S, Macrez R, Docagne F, Defer G, Laurent S, Rahman M, et al. Therapeutic benefits from nanoparticles: the potential significance of nanoscience in diseases with compromise to the blood brain barrier. Chem Rev 2012;113:1877-903.

65. Cao C, Wang X, Cai Y, Sun L, Tian L, Wu H, et al. Targeted in vivo imaging of microscopic tumors with ferritin-based nanoprobes across biological barriers. Adv Mater 2014;26:2566-71.

66. Krol S, Macrez R, Docagne F, Defer G, Laurent S, Rahman M, et al. Therapeutic benefits from nanoparticles: the potential significance of nanoscience in diseases with compromise to the blood brain barrier. Chem Rev 2013;113:1877-903.

67. Varallyay P, Nesbit G, Muldoon LL, Nixon RR, Delashaw J, Cohen JI, et al. Comparison of two superparamagnetic viral-sized iron oxide particles ferumoxides and ferumoxtran-10 with a gadolinium chelate in imaging intracranial tumors. Am J Neuroradiol 2002;23:510-9.

68. Neuwelt E, Varallyay P, Bago A, Muldoon L, Nesbit G, Nixon R. Imaging of iron oxide nanoparticles by MR and light microscopy in patients with malignant brain tumours. Neuropathol Appl Neurobiol 2004;30:456-71.

69. Manninger SP, Muldoon LL, Nesbit G, Murillo T, Jacobs PM, Neuwelt EA. An exploratory study of ferumoxtran-10 nanoparticles as a bloodbrain barrier imaging agent targeting phagocytic cells in CNS inflammatory lesions. Am J Neuroradiol 2005;26:2290-300.

70. Huse JT, Holland EC. Targeting brain cancer: advances in the molecular pathology of malignant glioma and medulloblastoma. Nat Rev Cancer 2010;10:319-31.

71. Giese A, Westphal M. Treatment of malignant glioma: a problem beyond the margins of resection. J Cancer Res Clin Oncol 2001;127:217-25.

72. Lyons SA, O’Neal J, Sontheimer H. Chlorotoxin, a scorpion-derived peptide, specifically binds to gliomas and tumors of neuroectodermal origin. Glia 2002;39:162-73.

73. Soroceanu L, Gillespie Y, Khazaeli M, Sontheimer H. Use of chlorotoxin for targeting of primary brain tumors. Cancer Res 1998;58:4871-9.

74. Meng X-x, Wan J-q, Jing M, Zhao S-g, Cai W, Liu E-z. Specific targeting of gliomas with multifunctional superparamagnetic iron oxide nanoparticle optical and magnetic resonance imaging contrast agents. Acta Pharmacol Sin 2007;28:2019.

75. Sun C, Veiseh O, Gunn J, Fang C, Hansen S, Lee D, et al. In vivo MRI detection of gliomas by chlorotoxin-conjugated superparamagnetic nanoprobes. Small 2008;4:372-9.

76. Shevtsov MA, Nikolaev BP, Ryzhov VA, Yakovleva LY, Dobrodumov AV, Marchenko YY, et al. Brain tumor magnetic targeting and biodistribution of superparamagnetic iron oxide nanoparticles linked with $70-\mathrm{kDa}$ heat shock protein study by nonlinear longitudinal response. J Magn Magn Mater 2015;388:123-34.

77. Wagner E, Curiel D, Cotten M. Delivery of drugs, proteins and genes into cells using transferrin as a ligand for receptor-mediated endocytosis. Adv Drug Deliv Rev 1994;14:113-35.

78. Ciechanover A, Schwartz A, Dautry-Varsat A, Lodish H. Kinetics of internalization and recycling of transferrin and the transferrin receptor in a human hepatoma cell line. Effect of lysosomotropic agents. J Biol Chem 1983;258:9681-9.
79. Durgadas C, Sharma CP, Sreenivasan K. Fluorescent and superparamagnetic hybrid quantum clusters for magnetic separation and imaging of cancer cells from blood. Nanoscale 2011;3:4780-7.

80. Jiang L, Zhou Q, Mu K, Xie H, Zhu Y, Zhu W, et al. pH/temperature sensitive magnetic nanogels conjugated with Cy5. 5-labeled lactoferrin for MR and fluorescence imaging of glioma in rats. Biomaterials 2013;34:7418-28.

81. Jansen M, Yip S, Louis DN. Molecular pathology in adult gliomas: diagnostic, prognostic, and predictive markers. Lancet Neurol 2010;9:717-26.

82. Shevtsov MA, Nikolaev BP, Yakovleva LY, Marchenko YY, Dobrodumov AV, Mikhrina AL, et al. superparamagnetic iron oxide nanoparticles conjugated with epidermal growth factor (sPION-egF) for targeting brain tumors. Int J Nanomedicine 2014;9:273.

83. Kohler N, Sun C, Fichtenholtz A, Gunn J, Fang C, Zhang M. Methotrexate-immobilized poly (ethylene glycol) magnetic nanoparticles for MR imaging and drug delivery. Small 2006;2:785-92.

84. Sun C, Sze R, Zhang M. Folic acid-PEG conjugated superparamagnetic nanoparticles for targeted cellular uptake and detection by MRI. $J$ Biomed Mater Res A 2006;78:550-7.

85. Kohler N, Sun C, Wang J, Zhang M. Methotrexate-modified superparamagnetic nanoparticles and their intracellular uptake into human cancer cells. Langmuir 2005;21:8858-64.

86. Leuschner C, Kumar CS, Hansel W, Soboyejo W, Zhou J, Hormes J. LHRH-conjugated magnetic iron oxide nanoparticles for detection of breast cancer metastases. Breast Cancer Res Treat 2006;99:163-76.

87. Chen T-J, Cheng T-H, Chen C-Y, Hsu SC, Cheng T-L, Liu G-C, et al. Targeted herceptin-dextran iron oxide nanoparticles for noninvasive imaging of HER2/neu receptors using MRI. JBIC J Biol Inorg Chem 2009;14:253-60.

88. Artemov D, Mori N, Okollie B, Bhujwalla ZM. MR molecular imaging of the Her-2/neu receptor in breast cancer cells using targeted iron oxide nanoparticles. Magn Reson Med 2003;49:403-8.

89. Huh Y-M, Y-w Jun, Song H-T, Kim S, Choi J-s, Lee J-H, et al. In vivo magnetic resonance detection of cancer by using multifunctional magnetic nanocrystals. J Am Chem Soc 2005;127:12387-91.

90. Kievit FM, Stephen ZR, Veiseh O, Arami H, Wang T, Lai VP, et al. Targeting of primary breast cancers and metastases in a transgenic mouse model using rationally designed multifunctional SPIONs. ACS Nano 2012;6:2591-601.

91. Kresse M, Wagner S, Pfefferer D, Lawaczeck R, Elste V, Semmler W. Targeting of ultrasmall superparamagnetic iron oxide (USPIO) particles to tumor cells in vivo by using transferrin receptor pathways. Magn Reson Med 1998;40:236-42.

92. Sipkins DA, Cheresh DA, Kazemi MR, Nevin LM, Bednarski MD, Li KC. Detection of tumor angiogenesis in vivo by $\alpha \mathrm{v} \beta 3$-targeted magnetic resonance imaging. Nat Med 1998;4:623-6.

93. Zhang C, Jugold M, Woenne EC, Lammers T, Morgenstern B, Mueller MM, et al. Specific targeting of tumor angiogenesis by RGD-conjugated ultrasmall superparamagnetic iron oxide particles using a clinical 1.5-T magnetic resonance scanner. Cancer Res 2007;67:1555-62.

94. Pasqualini R, Koivunen E, Ruoslahti E. av Integrins as receptors for tumor targeting by circulating ligands. Nat Biotechnol 1997;15:542.

95. Brooks PC, Clark R, Cheresh DA. Requirement of vascular integrin alpha v beta 3 for angiogenesis. Science 1994;264:569-71.

96. Montet X, Montet-Abou K, Reynolds F, Weissleder R, Josephson L. Nanoparticle imaging of integrins on tumor cells. Neoplasia 2006;8:214-22.

97. Xie J, Chen K, Lee H-Y, Xu C, Hsu AR, Peng S, et al. Ultrasmall c (RGDyK)-coated $\mathrm{Fe} 3 \mathrm{O} 4$ nanoparticles and their specific targeting to integrin $\alpha \mathrm{v} \beta 3$-rich tumor cells. J Am Chem Soc 2008;130:7542-3.

98. Yan C, Wu Y, Feng J, Chen W, Liu X, Hao P, et al. Anti- $\alpha v \beta 3$ antibody guided three-step pretargeting approach using magnetoliposomes for molecular magnetic resonance imaging of breast cancer angiogenesis. Int J Nanomedicine 2013;8:245. 
99. Huang J, Wang L, Lin R, Wang AY, Yang L, Kuang M, et al. Caseincoated iron oxide nanoparticles for high MRI contrast enhancement and efficient cell targeting. ACS Appl Mater Interfaces 2013;5:4632-9.

100. Gong F-m, Zhang Z-q, Chen X-d, Zhang L, Yu X-s, Yang Q-h, et al. A dual ligand targeted nanoprobe with high MRI sensitivity for diagnosis of breast cancer. Chin J Polym Sci 2014;32:321-32.

101. Foy SP, Manthe RL, Foy ST, Dimitrijevic S, Krishnamurthy N, Labhasetwar V. Optical imaging and magnetic field targeting of magnetic nanoparticles in tumors. ACS Nano 2010;4:5217-24.

102. Williams B, Alexander CM, Lindvall C, Mcconnell N. Mammary stem cell marker. US Patent 20,070,280,948; 2007.

103. Williams BO, Lindvall C. Low-density lipoprotein receptor 6 (LRP6) as a mammary stem cell marker and related methods. Google Patents; 2009.

104. Hess KR, Varadhachary GR, Taylor SH, Wei W, Raber MN, Lenzi R, et al. Metastatic patterns in adenocarcinoma. Cancer 2006;106:1624-33.

105. Hatabu H, Alsop DC, Listerud J, Bonnet M, Gefter WB. T2* and proton density measurement of normal human lung parenchyma using submillisecond echo time gradient echo magnetic resonance imaging. Eur J Radiol 1999;29:245-52.

106. Kuethe DO, Adolphi NL, Fukushima E. Short data-acquisition times improve projection images of lung tissue. Magn Reson Med 2007;57:1058-64.

107. Branca RT, Cleveland ZI, Fubara B, Kumar CS, Maronpot RR, Leuschner $\mathrm{C}$, et al. Molecular MRI for sensitive and specific detection of lung metastases. Proc Natl Acad Sci 2010;107:3693-7.

108. Jiang T, Zhang C, Zheng X, Xu X, Xie X, Liu H, et al. Noninvasively characterizing the different $\alpha v \beta 3$ expression patterns in lung cancers with RgD-UsPIO using a clinical $3.0 \mathrm{~T}$ MR scanner. Int $J$ Nanomedicine 2009;4:241.

109. Zhang C, Xie X, Liang S, Li M, Liu Y, Gu H. Mono-dispersed high magnetic resonance sensitive magnetite nanocluster probe for detection of nascent tumors by magnetic resonance molecular imaging. Nanomedicine 2012;8:996-1006.

110. Levin B, Lieberman DA, McFarland B, Smith RA, Brooks D, Andrews $\mathrm{KS}$, et al. Screening and surveillance for the early detection of colorectal cancer and adenomatous polyps, 2008: a joint guideline from the American Cancer Society, the US Multi-Society Task Force on Colorectal Cancer, and the American College of Radiology*†. $C A$ Cancer J Clin 2008;58:130-60.

111. Toma A, Otsuji E, Kuriu Y, Okamoto K, Ichikawa D, Hagiwara A, et al. Monoclonal antibody A7-superparamagnetic iron oxide as contrast agent of MR imaging of rectal carcinoma. Br J Cancer 2005;93:131-6.

112. Kitamura K, Miyagaki T, Yamaoka N, Tsurumi H, Noguchi A, Yamaguchi $\mathrm{T}$, et al. The role of monoclonal antibody A7 as a drug modifier in cancer therapy. Cancer Immunol Immunother 1993;36:177-84.

113. He X, Liu F, Liu L, Duan T, Zhang H, Wang Z. Lectin-conjugated Fe2O3@Aucore@ shell nanoparticles as dual mode contrast agents for in vivo detection of tumor. Mol Pharm 2014;11:738-45.

114. Jiang X, Housni A, Gody G, Boullanger P, Charreyre MT, Delair T, et al. Synthesis of biotinylated $\alpha$-d-mannoside or $\mathrm{N}$-acetyl $\beta$-d-glucosaminoside decorated gold nanoparticles: study of their biomolecular recognition with Con A and WGA lectins. Bioconjug Chem 2010;21:521-30.

115. Kirui DK, Khalidov I, Wang Y, Batt CA. Targeted near-IR hybrid magnetic nanoparticles for in vivo cancer therapy and imaging. $\mathrm{Na}$ nomedicine 2013;9:702-11.

116. Deckert P, Renner C, Cohen L, Jungbluth A, Ritter G, Bertino J, et al. A33scFv-cytosine deaminase: a recombinant protein construct for antibody-directed enzyme-prodrug therapy. Br J Cancer 2003;88:937-9.

117. Zou P, Yu Y, Wang YA, Zhong Y, Welton A, Galbán C, et al. Superparamagnetic iron oxide nanotheranostics for targeted cancer cell imaging and $\mathrm{pH}-\mathrm{dependent}$ intracellular drug release. Mol Pharm 2010;7:1974-84.

118. Farrera-Sinfreu J, Giralt E, Castel S, Albericio F, Royo M. Cell-penetrating cis- $\gamma$-amino-1-proline-derived peptides. J Am Chem Soc 2005;127:9459-68.
119. Cavalli S, Carbajo D, Acosta M, Lope-Piedrafita S, Candiota AP, Arús C, et al. Efficient $\gamma$-amino-proline-derived cell penetrating peptidesuperparamagnetic iron oxide nanoparticle conjugates via anilinecatalyzed oxime chemistry as bimodal imaging nanoagents. Chem Coтmun 2012;48:5322-4

120. Yu M, Jambhrunkar S, Thorn P, Chen J, Gu W, Yu C. Hyaluronic acid modified mesoporous silica nanoparticles for targeted drug delivery to CD44-overexpressing cancer cells. Nanoscale 2013;5:178-83.

121. Li J, He Y, Sun W, Luo Y, Cai H, Pan Y, et al. Hyaluronic acidmodified hydrothermally synthesized iron oxide nanoparticles for targeted tumor MR imaging. Biomaterials 2014;35:3666-77.

122. Liao Z, Wang H, Lv R, Zhao P, Sun X, Wang S, et al. Polymeric liposomes-coated superparamagnetic iron oxide nanoparticles as contrast agent for targeted magnetic resonance imaging of cancer cells. Langmuir 2011;27:3100-5.

123. Shahbazi-Gahrouei D, Abdolahi M. Superparamagnetic iron oxideC595: potential MR imaging contrast agents for ovarian cancer detection. J Med Phys Assoc Med Physicists India 2013;38:198.

124. Shahbazi-Gahrouei D, Abdolahi M. Detection of MUC1-expressing ovarian cancer by C595 monoclonal antibody-conjugated SPIONs using MR imaging. Sci World J 2013;2013.

125. Gao J, Chen K, Miao Z, Ren G, Chen X, Gambhir SS, et al. Affibodybased nanoprobes for HER2-expressing cell and tumor imaging. Biomaterials 2011;32:2141-8.

126. Lutz AM, Bachawal SV, Drescher CW, Pysz MA, Willmann JK, Gambhir SS. Ultrasound molecular imaging in a human CD276 expressionmodulated murine ovarian cancer model. Clin Cancer Res 2014;20:1313-22.

127. Welsh JB, Zarrinkar PP, Sapinoso LM, Kern SG, Behling CA, Monk $\mathrm{BJ}$, et al. Analysis of gene expression profiles in normal and neoplastic ovarian tissue samples identifies candidate molecular markers of epithelial ovarian cancer. Proc Natl Acad Sci 2001;98:1176-81.

128. Suwa T, Ozawa S, Ueda M, Ando N, Kitajima M. Magnetic resonance imaging of esophageal squamous cell carcinoma using magnetite particles coated with anti-epidermal growth factor receptor antibody. Int J Cancer 1998;75:626-34.

129. El-Boubbou K, Zhu DC, Vasileiou C, Borhan B, Prosperi D, Li W, et al. Magnetic glyco-nanoparticles: a tool to detect, differentiate, and unlock the glyco-codes of cancer via magnetic resonance imaging. $J$ Am Chem Soc 2010;132:4490-9.

130. Pilobello KT, Mahal LK. Deciphering the glycocode: the complexity and analytical challenge of glycomics. Curr Opin Chem Biol 2007;11:300-5.

131. Herr JK, Smith JE, Medley CD, Shangguan D, Tan W. Aptamerconjugated nanoparticles for selective collection and detection of cancer cells. Anal Chem 2006;78:2918-24.

132. Bhana S, Wang Y, Huang X. Nanotechnology for enrichment and detection of circulating tumor cells. Nanomedicine 2015;10:1973-90.

133. Vu-Quang H, Yoo M-K, Jeong H-J, Lee H-J, Muthiah M, Rhee JH, et al. Targeted delivery of mannan-coated superparamagnetic iron oxide nanoparticles to antigen-presenting cells for magnetic resonance-based diagnosis of metastatic lymph nodes in vivo. Acta Biomater 2011;7:3935-45.

134. Lim S-W, Kim H-W, Jun H-Y, Park S-H, Yoon K-H, Kim H-S, et al. TCL-SPION-enhanced MRI for the detection of lymph node metastasis in murine experimental model. Acad Radiol 2011;18:504-11.

135. Vu-Quang H, Muthiah M, Lee HJ, Kim Y-K, Rhee JH, Lee J-H, et al. Immune cell-specific delivery of beta-glucan-coated iron oxide nanoparticles for diagnosing liver metastasis by MR imaging. Carbohydr Polym 2012;87:1159-68.

136. Peiris PM, Toy R, Doolittle E, Pansky J, Abramowski A, Tam M, et al. Imaging metastasis using an integrin-targeting chain-shaped nanoparticle. ACS Nano 2012;6:8783-95.

137. Ocak M, Gillman AG, Bresee J, Zhang L, Vlad AM, Müller C, et al. Folate receptor-targeted multimodality imaging of ovarian cancer in a novel syngeneic mouse model. Mol Pharm 2015;12:542-53.

138. Sroka IC, Pond GD, Nagle RB, Porreca F, King T, Pestano G, et al. Human cell surface receptors as molecular imaging candidates for metastatic prostate cancer. Open Prostate Cancer J 2009;2:59. 
139. Mirshafiee V, Kim R, Park S, Mahmoudi M, Kraft ML. Impact of protein pre-coating on the protein corona composition and nanoparticle cellular uptake. Biomaterials 2016;75:295-304.

140. Mahmoudi M, Lohse SE, Murphy CJ, Fathizadeh A, Montazeri A, Suslick KS. Variation of protein corona composition of gold nanoparticles following plasmonic heating. Nano Lett 2014;14:6-12.

141. Mahmoudi M, Lynch I, Ejtehadi MR, Monopoli MP, Bombelli FB, Laurent S. Protein-nanoparticle interactions: opportunities and challenges. Chem Rev 2011;111:5610-37.

142. Casals E, Pfaller T, Duschl A, Oostingh GJ, Puntes V. Time evolution of the nanoparticle protein corona. ACS Nano 2010;4:3623-32.

143. Lundqvist M, Stigler J, Elia G, Lynch I, Cedervall T, Dawson KA. Nanoparticle size and surface properties determine the protein corona with possible implications for biological impacts. Proc Natl Acad Sci 2008; 105:14265-70.

144. Mahmoudi M, Simchi A, Milani A, Stroeve P. Cell toxicity of superparamagnetic iron oxide nanoparticles. $J$ Colloid Interface Sci 2009;336:510-8.

145. Cedervall T, Lynch I, Lindman S, Berggård T, Thulin E, Nilsson H, et al. Understanding the nanoparticle-protein corona using methods to quantify exchange rates and affinities of proteins for nanoparticles. Proc Natl Acad Sci 2007;104:2050-5.

146. Ghavami M, Saffar S, Emamy BA, Peirovi A, Shokrgozar MA, Serpooshan V, et al. Plasma concentration gradient influences the protein corona decoration on nanoparticles. RSC Adv 2013;3:1119-26.

147. Mahmoudi M, Lohse SE, Murphy CJ, Fathizadeh A, Montazeri A, Suslick KS. Variation of protein corona composition of gold nanoparticles following plasmonic heating. Nano Lett 2013;14:6-12.

148. Mahmoudi M, Shokrgozar MA, Behzadi S. Slight temperature changes affect protein affinity and cellular uptake/toxicity of nanoparticles. Nanoscale 2013;5:3240-4.

149. Hajipour MJ, Raheb J, Akhavan O, Arjmand S, Mashinchian O, Rahman M, et al. Personalized disease-specific protein corona influences the therapeutic impact of graphene oxide. Nanoscale 2015;7:8978-94.

150. Hajipour MJ, Laurent S, Aghaie A, Rezaee F, Mahmoudi M. Personalized protein coronas: a "key" factor at the nanobiointerface. Biomater Sci 2014;2:1210-21.

151. Caracciolo G, Pozzi D, Capriotti AL, Cavaliere C, Piovesana S, Amenitsch H, et al. Lipid composition: a "key factor" for the rational manipulation of the liposome-protein corona by liposome design. RSC Adv 2015;5:5967-75.

152. Caracciolo G, Caputo D, Pozzi D, Colapicchioni V, Coppola R. Size and charge of nanoparticles following incubation with human plasma of healthy and pancreatic cancer patients. Colloids Surf B: Biointerfaces 2014;123:673-8.

153. Walczyk D, Bombelli FB, Monopoli MP, Lynch I, Dawson KA. What the cell "sees" in bionanoscience. J Am Chem Soc 2010;132:5761-8.

154. Salvati A, Pitek AS, Monopoli MP, Prapainop K, Bombelli FB, Hristov $\mathrm{DR}$, et al. Transferrin-functionalized nanoparticles lose their targeting capabilities when a biomolecule corona adsorbs on the surface. Nat Nanotechnol 2013;8:137-43.

155. Mirshafiee V, Mahmoudi M, Lou K, Cheng J, Kraft ML. Protein corona significantly reduces active targeting yield. Chem Commun 2013;49:2557-9.

156. Mahmoudi M, Sheibani S, Milani AS, Rezaee F, Gauberti M, Dinarvand R, et al. Crucial role of the protein corona for the specific targeting of nanoparticles. Nanomedicine 2015;10:215-26.

157. Moyano DF, Saha K, Prakash G, Yan B, Kong H, Yazdani M, et al. Fabrication of corona-free nanoparticles with tunable hydrophobicity. ACS Nano 2014;8:6748-55.

158. Amiri H, Bordonali L, Lascialfari A, Wan S, Monopoli MP, Lynch I, et al. Protein corona affects the relaxivity and MRI contrast efficiency of magnetic nanoparticles. Nanoscale 2013;5:8656-65.

159. Shang L, Wang Y, Jiang J, Dong S. pH-dependent protein conformational changes in albumin: gold nanoparticle bioconjugates: a spectroscopic study. Langmuir 2007;23:2714-21.
160. Mahmoudi M, Shokrgozar MA, Sardari S, Moghadam MK, Vali H, Laurent $\mathrm{S}$, et al. Irreversible changes in protein conformation due to interaction with superparamagnetic iron oxide nanoparticles. Nanoscale 2011;3:1127-38.

161. Wu W-h, Sun X, Yu Y-p, Hu J, Zhao L, Liu Q, et al. TiO2 nanoparticles promote $\beta$-amyloid fibrillation in vitro. Biochem Biophys Res Commun 2008;373:315-8.

162. Saei AA, Mahmoudi M. Toxicity of nanoparticles. Nanoparticles for delivery of biotherapeutics. Future Med 2014;1:112-31, http:// dx.doi.org/10.4155/fseb2013.14.74.

163. You C-C, Agasti SS, De M, Knapp MJ, Rotello VM. Modulation of the catalytic behavior of $\alpha$-chymotrypsin at monolayer-protected nanoparticle surfaces. J Am Chem Soc 2006;128:14612-8.

164. You C-C, De M, Rotello VM. Contrasting effects of exterior and interior hydrophobic moieties in the complexation of amino acid functionalized gold clusters with $\alpha$-chymotrypsin. Org Lett 2005;7:5685-8.

165. De M, Rotello VM. Synthetic "chaperones": nanoparticle-mediated refolding of thermally denatured proteins. Chem Commun 2008;3504-6.

166. Walkey CD, Olsen JB, Guo H, Emili A, Chan WC. Nanoparticle size and surface chemistry determine serum protein adsorption and macrophage uptake. J Am Chem Soc 2012;134:2139-47.

167. Nagayama S, Ogawara K, Fukuoka Y, Higaki K, Kimura T. Timedependent changes in opsonin amount associated on nanoparticles alter their hepatic uptake characteristics. Int J Pharm 2007;342:215-21.

168. Rubel C, Fernández GC, Dran G, Bompadre MB, Isturiz MA, Palermo MS. Fibrinogen promotes neutrophil activation and delays apoptosis. $J$ Immunol 2001;166:2002-10.

169. Sitrin RG, Pan PM, Srikanth S, Todd RF. Fibrinogen activates NF-kB transcription factors in mononuclear phagocytes. J Immunol 1998;161:1462-70.

170. Ruge CA, Kirch J, Cañadas O, Schneider M, Perez-Gil J, Schaefer UF, et al. Uptake of nanoparticles by alveolar macrophages is triggered by surfactant protein A. Nanomed Nanotechnol Biol Med 2011;7:690-3.

171. Saie AA, Ray M, Mahmoudi M, Rotello VM. Engineering the nanoparticle-protein interface for cancer therapeutics. Nanotechnology-based precision tools for the detection and treatment of cancer. Springer; 2015245-73.

172. Tenzer S, Docter D, Kuharev J, Musyanovych A, Fetz V, Hecht R, et al. Rapid formation of plasma protein corona critically affects nanoparticle pathophysiology. Nat Nanotechnol 2013;8:772-81.

173. Hu W, Peng C, Lv M, Li X, Zhang Y, Chen N, et al. Protein coronamediated mitigation of cytotoxicity of graphene oxide. ACS Nano 2011;5:3693-700.

174. Hamad I, Al-Hanbali O, Hunter AC, Rutt KJ, Andresen TL, Moghimi SM. Distinct polymer architecture mediates switching of complement activation pathways at the nanosphere-serum interface: implications for stealth nanoparticle engineering. ACS Nano 2010;4:6629-38

175. Kah JCY, Chen J, Zubieta A, Hamad-Schifferli K. Exploiting the protein corona around gold nanorods for loading and triggered release. ACS Nano 2012;6:6730-40.

176. Mahmoudi M, Abdelmonem AM, Behzadi S, Clement JH, Dutz S, Ejtehadi MR, et al. Temperature: the "ignored" factor at the NanoBio interface. ACS Nano 2013;7:6555-62.

177. Högemann-Savellano D, Bos E, Blondet C, Sato F, Abe T, Josephson $\mathrm{L}$, et al. The transferrin receptor: a potential molecular imaging marker for human cancer. Neoplasia 2003;5:495-506.

178. Srivastava RAK. Scavenger receptor class B type I expression in murine brain and regulation by estrogen and dietary cholesterol. $J$ Neurol Sci 2003;210:11-8.

179. Zannis VI, Chroni A, Krieger M. Role of apoA-I, ABCA1, LCAT, and SR-BI in the biogenesis of HDL. J Mol Med 2006;84:276-94.

180. Ahmed H, Blakeley M, Cianci M, Cruickshank D, Hubbard J, Helliwell $\mathrm{J}$. The determination of protonation states in proteins. Acta Crystallogr D Biol Crystallogr 2007;63:906-22. 